\title{
Turbulent velocity structure in molecular clouds
}

\author{
V. Ossenkopf ${ }^{1}$ and M.-M. Mac Low ${ }^{2}$ \\ 1 I. Physikalisches Institut, Universität zu Köln, Zülpicher Straße 77, 50937 Köln, Germany \\ 2 Department of Astrophysics, American Museum of Natural History, Central Park West at 79th Street, \\ New York, NY 10024-5192, USA
}

Received 13 December 2000 / Accepted 22 April 2002

\begin{abstract}
We compare velocity structure observed in the Polaris Flare molecular cloud at scales ranging from $0.015 \mathrm{pc}$ to $20 \mathrm{pc}$ to the velocity structure of a suite of simulations of supersonic hydrodynamic and MHD turbulence computed with the ZEUS MHD code. We examine different methods of characterising the structure, including a scanning-beam method that provides an objective measurement of Larson's size-linewidth relation, structure functions, velocity and velocity difference probability distribution functions (PDFs), and the $\Delta$-variance wavelet transform, and use them to compare models and observations.

The $\Delta$-variance is most sensitive to characteristic scales and scaling laws, but is limited in its application by a lack of intensity weighting so that its results are easily dominated by observational noise in maps with large empty areas. The scanning-beam size-linewidth relation is more robust with respect to noisy data. Obtaining the global velocity scaling behaviour requires that large-scale trends in the maps not be removed but treated as part of the turbulent cascade. We compare the true velocity PDF in our models to simulated observations of velocity centroids and average line profiles in optically thin lines, and find that the line profiles reflect the true PDF better unless the map size is comparable to the total line-of-sight thickness of the cloud. Comparison of line profiles to velocity centroid PDFs can thus be used to measure the line-of-sight depth of a cloud.

The observed density and velocity structure is consistent with supersonic turbulence with a driving scale at or above the size of the molecular cloud and dissipative processes below $0.05 \mathrm{pc}$. Ambipolar diffusion could explain the dissipation. Over most of the observed range of scales the velocity structure is that of a shock-dominated medium driven from large scale. The velocity PDFs exclude small-scale driving such as that from stellar outflows as a dominant process in the observed region. In the models, large-scale driving is the only process that produces deviations from a Gaussian PDF shape consistent with observations, almost independent of the strength of driving or magnetic field. Strong magnetic fields impose a clear anisotropy on the velocity field, reducing the velocity variance in directions perpendicular to the field.
\end{abstract}

Key words. ISM: clouds - ISM: magnetic fields, turbulence - ISM: kinematics and dynamics - MHD

\section{Introduction}

Attempts to characterise the physical state of molecular clouds by comparison to simulations must rely on statistical descriptions of the observations and the simulations. In the last decade, several techniques have been used to characterise the observed radial velocity distribution in representative molecular clouds, as reviewed by Goodman et al. (1998) and Miesch et al. (1999).

First attempts to characterise the scaling behaviour of the velocity structure started from one of the famous "Larson's laws" (Larson 1981), showing a power law relation between the size and the linewidth measured for a molecular cloud. This relation has been extended from integrated velocities to velocity fluctuations within clouds by Miesch \& Bally (1994), providing similar power laws. On the other hand hydrodynamic and magnetohydrodynamic (MHD) simulations have been traditionally characterised using the probability distribution functions (PDFs) of velocities and velocity differences

Send offprint requests to: V. Ossenkopf, e-mail: ossk@ph1.uni-koeln.de (e.g. Anselmet et al. 1984). Both approaches have been unified by Lis et al. (1996) and Miesch et al. (1999), who measured the PDFs of line centroid velocities and the scaling behaviour of PDFs of centroid velocity differences as a function of lag for several star-forming clouds.

Mac Low \& Ossenkopf (2000, hereafter Paper I) used the $\Delta$-variance, a multi-dimensional wavelet transform (Stutzki et al. 1998), to characterise both the density and the velocity structure of interstellar turbulence simulations. For the density structure, a direct comparison to the analysis of observed clouds provided by Bensch et al. (2001a) was possible. In velocity space there is no direct observational measure for the $\Delta$-variance. We need additional tools for the quantification of the turbulent velocity structure that can be determined with the same ease for observations and simulations, and containing at least as much information as the $\Delta$-variance.

In this paper we test five different methods on an observational data set covering three steps of angular resolution in the Polaris Flare, a translucent molecular cloud, and on a number of gas dynamical and MHD simulations of interstellar 
turbulence. The first two of the methods characterise the total velocity distribution: the PDFs of the total velocity distribution and of the line centroid velocities. The other three methods characterise the spatial distribution of velocities: a generalised size-linewidth ("Larson") relation, the dependence of the low order moments of the centroid velocity difference PDF on lag, and the $\Delta$-variance analysis.

Comparisons between observations and models have been made with a simulation of mildly supersonic, decaying hydrodynamic turbulence (Falgarone et al. 1991; Falgarone et al. 1994; Falgarone et al. 1995; Falgarone et al. 1995; Lis et al. 1996; Lis et al. 1998; Joulain et al. 1998; and Pety \& Falgarone 2000), with MHD models of supersonic turbulence neglecting self-gravity by Padoan et al. (1998), Padoan et al. (1999) and Padoan et al. (2000), with decaying and driven self-gravitating, hydrodynamic turbulence (Klessen 2000), and with various ad hoc models of turbulence (e.g. Dubinski et al. 1995; Chappell \& Scalo 1999). Observations suggest that supersonic, super-Alfvénic turbulence is an appropriate physical model (see Padoan \& Nordlund 1999). Here, we want to test whether it can reproduce the observed velocity structure and find what we can learn about the physical conditions of turbulent molecular clouds from comparison to a large set of models computed by Mac Low et al. (1998) and Mac Low (1999).

In Sect. 2 we discuss the Polaris Flare observational data used here and the basic data processing applied. In Sect. 3 the different tools for the analysis of the velocity structure are introduced and applied to the observational data. The turbulence simulations used for comparison are presented in Sect. 4 and the results of the velocity analysis for these models are given in Sect. 5. Section 6 concludes with a discussion on the physical state of the cloud based on our comparisons.

\section{Observational data}

Molecular line observations only determine line profiles, which give the convolution of the radial velocity component with density along the line of sight. The situation becomes even more complicated if one takes optical depth effects and spatially varying temperatures and excitation levels into account. For the analysis provided here we restrict ourselves to the assumption of constant excitation conditions in an optically thin medium, so that the integrated line intensity is a direct measure of the column density.

\subsection{Polaris Flare observations}

In Paper I, we compared our analysis of simulations with an analysis of the intensity structure of multiscale observations of the Polaris Flare performed by Bensch et al. (2001a). As no prior analysis of the velocity structure observed in this data has been done, we present that here as one point of comparison to the simulation results.

For the velocity field analysis we use three of the observational data sets studied by Bensch et al. (2001a) in the analysis of the intensity structure. The Polaris Flare observations consist of a set of nested maps obtained with the $1.2 \mathrm{~m} \mathrm{CfA}$ telescope, the $3 \mathrm{~m}$ KOSMA, and the $30 \mathrm{~m}$ IRAM. The CfA data were taken in ${ }^{12} \mathrm{CO} 1-0$ at a spatial resolution (HPBW) of 8.7' (Heithausen \& Thaddeus 1990); the KOSMA observations used the ${ }^{12} \mathrm{CO} 2-1$ transition at 2.2' resolution (Bensch et al. 2001a); and IRAM observations of the MCLD 123.5+24.9 region in the Polaris Flare were taken in the two lower transitions of ${ }^{12} \mathrm{CO}$ and ${ }^{13} \mathrm{CO}$ and in the $1-0$ transition of $\mathrm{C}^{18} \mathrm{O}$ within the IRAM key-project "Small-scale structure of pre-star-forming regions" (Falgarone et al. 1998). To discuss a consistent set of observations for all maps we restrict ourselves to the ${ }^{12} \mathrm{CO}$ IRAM data. Because of the higher signal-to-noise ratio, we only use the $1-0$ lines taken at $0.35^{\prime}$ resolution. Assuming a distance to the Polaris Flare cloud of $150 \mathrm{pc}^{1}$, the telescope resolutions translate into physical resolutions of $0.38 \mathrm{pc}, 0.09 \mathrm{pc}$, and $0.015 \mathrm{pc}$ respectively. Altogether these observations provide a data set covering more than three decades in linear resolution from 0.015 pc to about $50 \mathrm{pc}$.

A major problem when combining these data is the different channel width, noise, and baseline behaviour of the different instruments and observational runs. The IRAM observations show an rms noise of $0.5 \mathrm{~K}$ at $0.05 \mathrm{~km} \mathrm{~s}^{-1}$, with some large scale trends in the noise indicating that either the spectral or the spatial baseline is not optimal. The KOSMA data have an rms of $0.4 \mathrm{~K}$ at $0.05 \mathrm{~km} \mathrm{~s}^{-1}$ throughout the whole map. The CfA data show an rms noise of $0.1 \mathrm{~K}$ at $0.65 \mathrm{~km} \mathrm{~s}^{-1}$, and show both a variation of the absolute noise level throughout the map, and some areas with slightly negative intensities, indicating imperfect baselines. This opens up some uncertainties when combining noise sensitive results from the three data cubes.

\subsection{Line windowing}

The basic problem in the deduction of the velocity structure is the finite signal-to-noise ratio in each observed map, often combined with an imperfect spectral baseline. Due to small slopes and variations of the baselines, and a slightly variable noise throughout the spectrum, the determination of the velocity structure from the spectra is sensitive to the exact selection of the spectral window around the line considered. The influence of this effect increases from the line centroid velocities to the higher moments like the variances or the kurtosis. A detailed discussion of these problems in the determination of the centroid velocity was provided by Miesch \& Bally (1994) and Miesch \& Scalo (1995).

We have tested three different methods for the selection of that part of the spectrum containing as much information as possible about the line but least influenced by baseline uncertainties. First we applied a global windowing technique defining a minimum velocity range covering all noticeable emission. We used this global windowing as a first rough constraint to the velocity space, including all channels where eye inspection might still guess some line contribution. A second method that we have extensively tested was to search for the line contribution interval by using the first zeros at the flanks of the lines. We found, however, that too many lines are either relatively weak or break up into several components so that zeros occur

\footnotetext{
Heithausen \& Thaddeus (1990) estimated a distance to the Polaris Flare cloud of $240 \mathrm{pc}$ whereas Zagury et al. (1999) derived 105$125 \mathrm{pc}$.
} 
within the line. Even the centroid velocities could not be reliably determined from this approach. As a third approach, we have used a typical criterion for noticeable emission, such as emission above a $3 \sigma$ noise level. It turned out, however, that for most values of the significance level, we clearly miss part of the information from weak but broad lines in thin outer regions that inspection by eye would still count as a part of the line.

Thus we show for all our observational results large error bars given by two extremes of this criterion: we count either all contributions within the global spectral window, or only channels above the maximum noise level given by the largest negative value. As representative intermediate values we show in all plots the results using all positive contributions above $1 \sigma$ of the noise. This third level provides a reasonable intermediate value and the parameters from the data analysis below show only a small variation when changing the noise cut level around this value. One should however keep in mind that we do not know the best treatment of the noise so that this line should be considered only as guiding the eye but not as the best representative or average value.

\subsection{Large-scale trends}

Miesch \& Bally (1994) extensively discussed the removal of large scale trends in the centroid velocity maps to get a significant description of the turbulent velocity fluctuations undisturbed by any large systematic motions. We will not follow their method here when dealing with the Polaris Flare data. Due to the nested nature of the different maps, any large scale motion on a smaller map is only a velocity fluctuation on the larger map. As Bensch et al. (2001a) have shown for the intensity maps, a smooth transition in the scaling behaviour of the three maps is only possible if large scale trends are not removed.

Furthermore, there is no clear separation between turbulent and systematic motions. Any assumed separation scale is arbitrary as long as there is no physical process such as energy injection at that scale. Removal of velocities at certain scales might prevent understanding of the underlying processes. Even the largest systematic motions, like Galactic rotation, may be part of the turbulent cascade if they inject energy into the system.

It could be justifiable to remove the large scale trends for the star-forming regions considered by Miesch \& Bally (1994) if the turbulence there were only driven by small-scale starformation activity, and not by large-scale motions. However, that is not proven even there, and we would certainly miss the main physics by applying the same kind of separation for the Polaris Flare data, where the turbulence probably is driven by motions on the largest scales (see discussion in Paper I).

\section{Statistical descriptions of velocity structure and their applicability to observations}

\subsection{Size-linewidth relation}

A traditional measure for the spatial velocity distribution is the size-linewidth relation for clouds identified by
Larson (1981) and obtained by many observers since then. To measure this, one has to define objects within a map, such as molecular clouds or clumps within clouds, and relate the effective linewidths measured for these objects to their characteristic sizes. This method has been used to study a large variety of clouds, clumps, and cores (e.g. Myers 1983; Caselli \& Myers 1995; Peng et al. 1998). A comprehensive recent overview including a careful estimate of many possible errors was given by Goodman et al. (1998). Most studies obtain power laws

$\Delta v_{\mathrm{obs}} \propto R^{\gamma} \quad$ with $\gamma=0.2 \ldots 0.7$

over wide spatial ranges, where $R$ denotes the effective radius of the object. A search for Larson-type relations in turbulence simulations was performed by Vázquez-Semadeni et al. (1997).

A major problem in the computation of these size-linewidth relations is the somewhat arbitrary definition of the objects in the observed position-velocity space that are considered to give definite values for sizes and linewidths. This definition is obvious, though dynamically arbitrary, for isolated molecular clouds, but difficult when selecting clumps within a cloud, and completely impractical for filamentary, turbulent cloud structures.

As an alternative, one can compute a size-linewidth relation by measuring the average linewidths within telescope beams of varying size - effectively the intensity-weighted velocity dispersion within a varying radius. Practically, the observed map is scanned with a Gaussian of varying size and the average linewidth is determined for each size. In averaging, each position is weighted by its total intensity so that the lower significance of "empty" regions is taken into account. This method can be easily applied to each data set and the results are directly comparable to the traditional size-linewidth relations.

We face, however, the problem that the velocity dispersion for each virtual beam depends not only on its size, but also on the cloud depth along the line of sight. This depth enters by changing the local linewidth at each point. To separate the two length scales we have applied the analysis both to the total velocity dispersion within the Gaussian, and to the dispersion of the line centroids observed at each point in the map. In the latter case, we hope to remove the influence of the cloud depth.

In Fig. 1 we show both relations for the Polaris Flare observations. The two extreme approaches for the noise treatment (taking all emission from the spectral window or only emission above the maximum noise level) produce relatively large error bars in the plots for both the total velocity dispersion and the centroid velocity dispersion. The CfA data with their low spectral resolution in particular have error bars of up to a factor of two. Within the errors, however, we find a unique smooth behaviour in both quantities from the smallest to the largest scales. The KOSMA data do deviate somewhat from the other two sets. This can be partially attributed to the different $\mathrm{CO}$ transition observed by that telescope. We have checked this effect by computing the same plot for the IRAM ${ }^{12} \mathrm{CO} 2-1$ data. Although the results were shifted relative to the IRAM 1-0 data in the same direction as the KOSMA data, they do not line up 


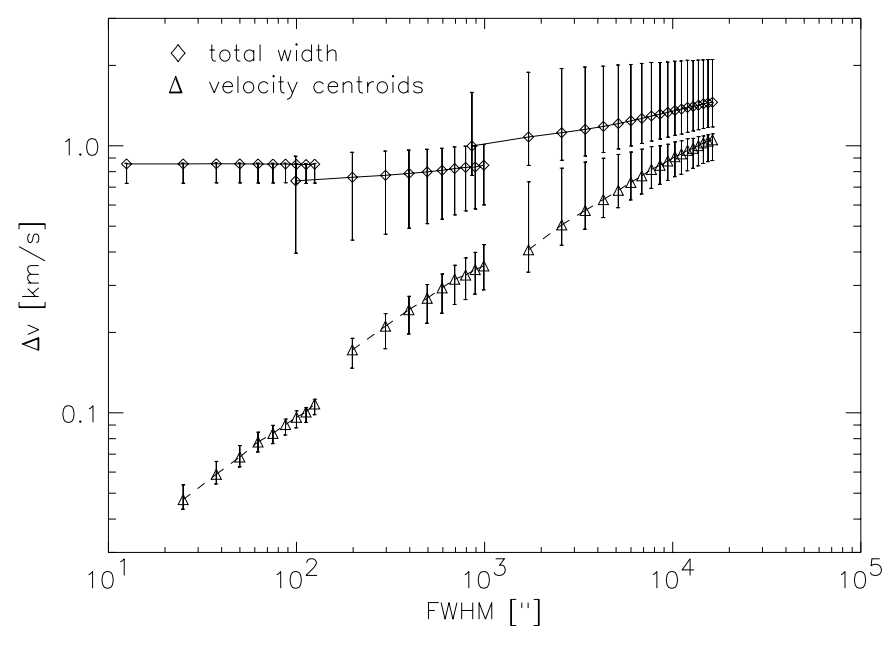

Fig. 1. Size-linewidth relation for the Polaris Flare CO observations with IRAM (smallest scales), KOSMA, and the CfA $1.2 \mathrm{~m}$ telescope (largest scale, see Bensch et al. (2001a) for details). The diamonds show the relation when the linewidth for a given scale is integrated from the total linewidths at each point. The triangles represent the widths when only the dispersions of the centroids of the lines are measured. The error bars do not represent true local errors but the two extreme cases of the line windowing as discussed in Sect. 2.2.

exactly with the KOSMA result. Thus the shift is probably also influenced by the different noise behaviour.

For the size-linewidth relation based on the velocity centroids, we find one power law stretching over three orders of magnitude connecting the three different maps. The average velocity variances range from below the thermal linewidth up to about $1 \mathrm{~km} \mathrm{~s}^{-1}$. The common slope is given by $\gamma=0.50 \pm 0.04$. However, the data are also consistent with a reduction of the slope down to 0.24 at the largest scales, if the full extent of the error bars is taken into account.

In the size-linewidth relationship integrated from the full local linewidths, there is a transition of the slope from almost zero at scales below $10^{\prime}$ to 0.2 at the full size of the flare. The plot shows that the total linewidths are dominated by the lineof-sight integration up to the largest scales.

Although the slopes measured with this method are very shallow, they do appear to show the change of slope interpreted by Goodman et al. (1998) as a transition to coherent behaviour below about $0.5 \mathrm{pc}$. As the findings of Goodman et al. are also based on the total linewidths this suggests that the change might rather reflect the transition from a regime where single separated clumps are identified, to measurements of a superposition of substructures at smaller scales.

\subsection{Velocity probability distribution function}

Another quantity characterising the velocity structure both in observational data and in turbulence simulations is the probability distribution function (PDF) of velocities. Although it contains no information on the spatial correlation in velocity space like the size-linewidth relation or the $\Delta$-variance, it shows complementary properties, like the degree of intermittency in the turbulent structure (Falgarone \& Phillips 1990).
The shape of the wings of the velocity PDF is thought to be diagnostic of intermittency, where the increasing degrees of intermittency produces a transition from Gaussian to exponential wings. Two-dimensional Burgers turbulence simulations by Chappell \& Scalo (1999), neglecting pressure forces, showed Gaussian velocity PDFs for models of decaying turbulence and exponential wings for models driven by strong stellar winds.

Due to the limited amount of information available from molecular lines, there is no direct way to deduce the velocity PDF from observations. One approach to deducing the velocity PDFs is computation of the distribution of line centroid velocities (Kleiner \& Dickmann 1985; Miesch \& Bally 1994; Miesch et al. 1999). This method can also include some information on spatial correlation as discussed in Sect. 3.3. However, the higher moments of the centroid PDF are very sensitive to the observational restrictions discussed in Sect. 2.2.

Another method was introduced by Falgarone \& Phillips (1990), who estimated velocity PDFs from high signal-to-noise observations of single line profiles. Investigating the statistical moments of profiles, Falgarone et al. (1994) found no simple Gaussian behaviour for many observations and provided a first comparison with three-dimensional (3D) hydrodynamic simulations. Most of their PDFs could be represented by a superposition of two Gaussians where the wing component had about three times the width of the core component. Unfortunately, their method is only reliable for optically thin transitions at a very high signal to noise. We test both methods here, starting with the centroid velocity PDF.

\subsubsection{Centroid velocity PDFs}

In computing the centroid velocity PDF for a map one can either assign the same weight to each point in the map, or weight the different contributions by the intensities measured at that point. We find that the PDFs retain similar shape and the same wing behaviour with both methods, and therefore use intensity weighting in the following analysis, as it is less influenced by observational noise. We have also used normal histograms here, instead of the more sophisticated Johnson PDF estimator applied by Miesch et al. (1999) because the error bars present from the uncertainty about the noise treatment greatly exceed the influence of the numerical PDF estimator.

Figure 2 shows the centroid velocity PDFs for the three data sets. We find that the IRAM and the CfA data are characterised by an asymmetry of the velocity distribution, indicating some kind of large-scale flow within the mapped region. Looking at the wings of the distributions, however, all three data sets are consistent with a Gaussian, which would appear as a parabola in the lin-log plots shown. Only at the scale of the CfA map is a definite conclusion not possible, due to the large error bars.

Beyond this phenomenological approach, the shape of the PDFs can be quantified by their statistical moments. The most frequently used moments are

$$
\begin{aligned}
& \left\langle v_{\mathrm{c}}\right\rangle=\int_{-\infty}^{\infty} \mathrm{d} v_{\mathrm{c}} P\left(v_{\mathrm{c}}\right) v_{\mathrm{c}} \\
& \sigma^{2}=\int_{-\infty}^{\infty} \mathrm{d} v_{\mathrm{c}} P\left(v_{\mathrm{c}}\right)\left[v_{\mathrm{c}}-\left\langle v_{\mathrm{c}}\right\rangle\right]^{2}
\end{aligned}
$$



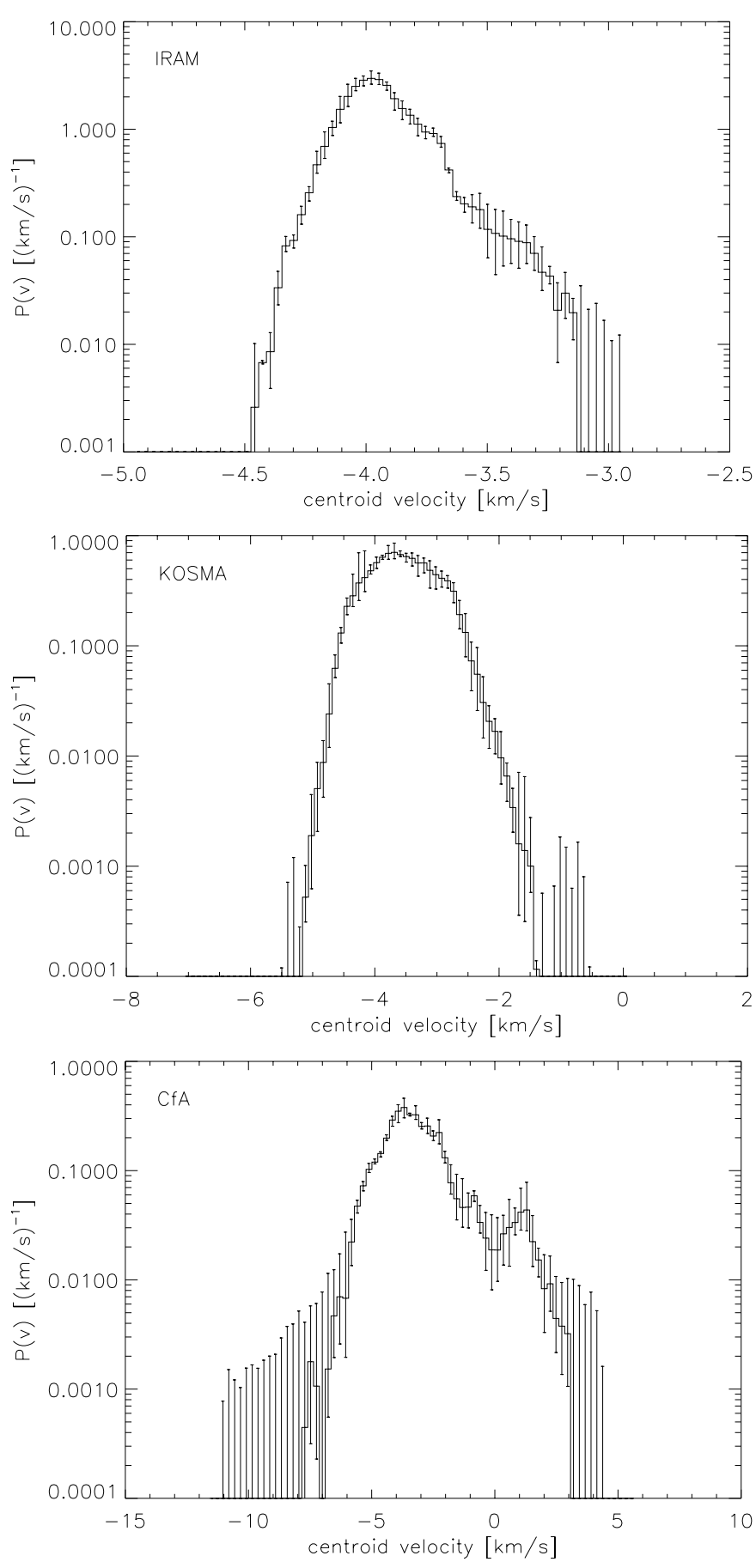

Fig. 2. Probability density distribution of centroid velocities for the three Polaris Flare data cubes. The error bars show the deviation introduced by different treatments of the observational noise, as discussed in the text.

$K=\frac{1}{\sigma^{4}} \int_{-\infty}^{\infty} \mathrm{d} v_{\mathrm{c}} P\left(v_{\mathrm{c}}\right)\left[v_{\mathrm{c}}-\left\langle v_{\mathrm{c}}\right\rangle\right]^{4}$

where $\left\langle v_{\mathrm{c}}\right\rangle$ is the mean, $\sigma^{2}$ the variance, and $K$ the kurtosis of the distribution. The probability distribution function $P$ is normalised to unity. The variance is a measure for the total turbulent mixing energy, while the kurtosis characterises the deviation from a Gaussian profile. It takes a value of three for a Gaussian distribution, and six for a distribution with exponen- tial wings. Values between about 2.7 and 3.0 are still consistent with a Gaussian which is truncated due to a finite sample size. Shallow wings become obvious for kurtosis values above about 3.4.

We restrict the analysis to these low moments because higher moments become increasingly uncertain due to the influence of observational noise, non-perfect spectral baselines, and error-beam pickup (see e.g. Bensch et al. 2001b). Without extremely high signal to noise data it is impossible to obtain reliable constraints on the spatial variation even for the next higher velocity moments. The situation is even worse for methods like the spectral correlation function (Rosolowsky et al. 1999) that include all details of the line profiles.

In Table 1 we give the standard deviation (square root of the variance) and kurtosis for the three PDFs. Only the KOSMA map shows an almost Gaussian distribution of velocity centroids with slightly steeper-than-Gaussian wings. The kurtosis for the other two maps is clearly larger than Gaussian. We have tested whether we can reproduce this by the superposition of two Gaussians as proposed by Falgarone et al. (1991). Within the error bars we always obtained good fits. All wings can be reproduced by a Gaussian. The widths of the core and the wing component obtained from this fit are also given in Table 1. In contrast to the width ratio of about three obtained by Falgarone et al. we find a ratio of about two.

Only a few of the observed maps analysed by Miesch et al. (1999) showed approximately Gaussian centroid velocity PDFs, while the majority had PDFs with shallower wings that could be fitted with either exponential laws or power laws. Fitting the exponent, Miesch et al. (1999) obtained two different values when treating either the whole distribution or only the wings. This is similar to our fit of the observational data with two different Gaussians for the wing and the core component.

In the Polaris Flare maps the wings of all distributions can be represented by Gaussians. However, the total distributions sometimes deviate considerably from Gaussian behaviour resulting in a Gaussian kurtosis value at intermediate scales (the KOSMA map) compared to significantly larger kurtosis at smaller and larger scales. Besides the effect of observational errors, this might represent the influence of systematic velocity trends across the mapped region which appear mainly in the core component but not in the wing. We don't expect these trends in the isotropic simulations analysed in Sect. 5.2.

\subsubsection{Average line profiles}

In Fig. 3 we show the average line profiles for all three data sets. The corresponding moments and fit parameters of the distributions are also given in Table 1. The average line profile automatically contains the weighting of each velocity contribution by its intensity as discussed above for the centroid PDFs, so that we can compare both.

The general shape is similar but the line profiles are much broader and the peak positions are not at the same velocity as in the PDFs. For a detailed comparison, we plot both the 
Table 1. Parameters of the centroid velocity PDFs and the average line profiles in the three Polaris Flare maps.

\begin{tabular}{llllll}
\hline \hline PDF type & telescope & $\begin{array}{l}\text { std. deviation } \\
{\left[\mathrm{km} \mathrm{s}^{-1}\right]}\end{array}$ & kurtosis & $\begin{array}{l}\text { core width }^{a} \\
{\left[\mathrm{~km} \mathrm{~s}^{-1}\right]}\end{array}$ & $\begin{array}{l}\text { wing width } \\
{\left[\mathrm{km} \mathrm{s}^{-1}\right]}\end{array}$ \\
\hline centroid PDFs & IRAM & $0.17 \pm 0.01$ & $5.1 \pm 0.2$ & $0.130 \pm 0.005$ & $0.24 \pm 0.05$ \\
& KOSMA & $0.53 \pm 0.03$ & $2.7 \pm 0.1$ & $0.57 \pm 0.05$ & $0.41 \pm 0.03$ \\
& CfA & $1.6 \pm 0.2$ & $5.0 \pm 0.3$ & $1.02 \pm 0.05$ & $2.2 \pm 0.5$ \\
line profiles & IRAM & 0.88 & 2.4 & $0.98 \pm 0.04$ & $0.80 \pm 0.07$ \\
& KOSMA & 1.06 & 2.7 & $1.10 \pm 0.01$ & $1.03 \pm 0.05$ \\
& CfA & 2.3 & 3.8 & $1.8 \pm 0.1$ & $2.7 \pm 0.3$ \\
\hline
\end{tabular}

${ }^{a}$ Standard deviation of the Gaussian fit.

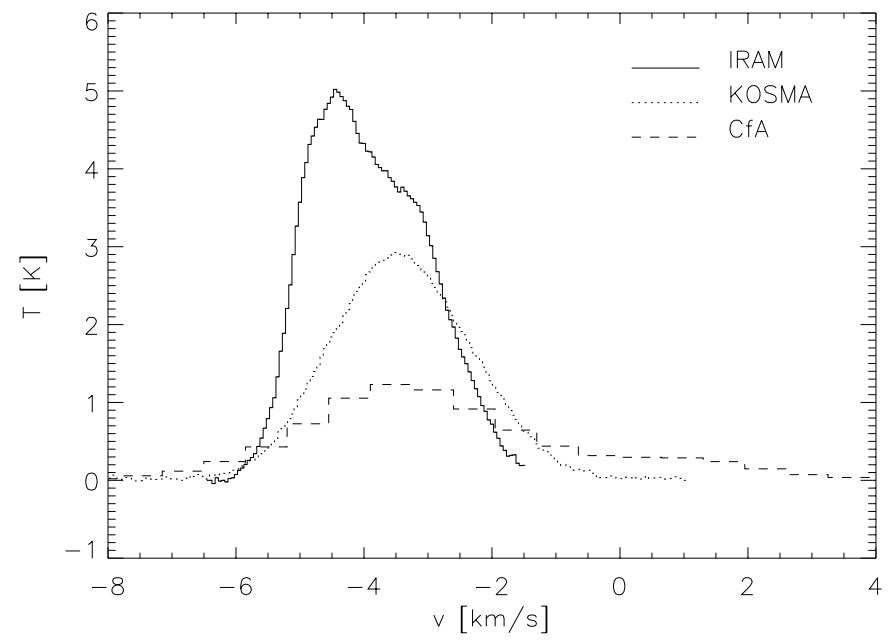

Fig. 3. Average line profiles for the three Polaris Flare data cubes. The solid line shows the IRAM data, the dotted line the average profile in the KOSMA map multiplied by 2 and the dashed line the CfA data multiplied by 8 .

average line profile and the centroid velocity distribution for the IRAM data on the same logarithmic scale in Fig. 4. The ratio between average line profile width and centroid velocity PDF width measured from the Gaussian fitted to the wings of the distributions is about 3.4 for the IRAM map, 2.2 for the KOSMA map, and 1.5 for the CfA map. Using the variance of the full distributions we get ratios of 5.2, 2.0, and 1.4 respectively for the three maps. The difference of the two ratios for the IRAM map corresponds to some large scale velocity flow on that scale producing the irregular PDF core seen in Fig. 4.

The variation of this ratio with the size of the map is again naturally explained by the two different length scales involved: the line-of-sight integration and the size of the map. In the IRAM data, the small size of the map provides a relatively narrow centroid velocity PDF compared to the broad average line profile determined by the line-of-sight integration through the full depth of the cloud. In contrast, the thickness of the cloud will certainly be smaller than the full extent of the CfA map. This is also indicated by the approaching slopes of the two different variances within beams of varying size in Fig. 1 at large scales. To compare the ratios obtained here with turbulence simulations in model cubes we have to consider scales

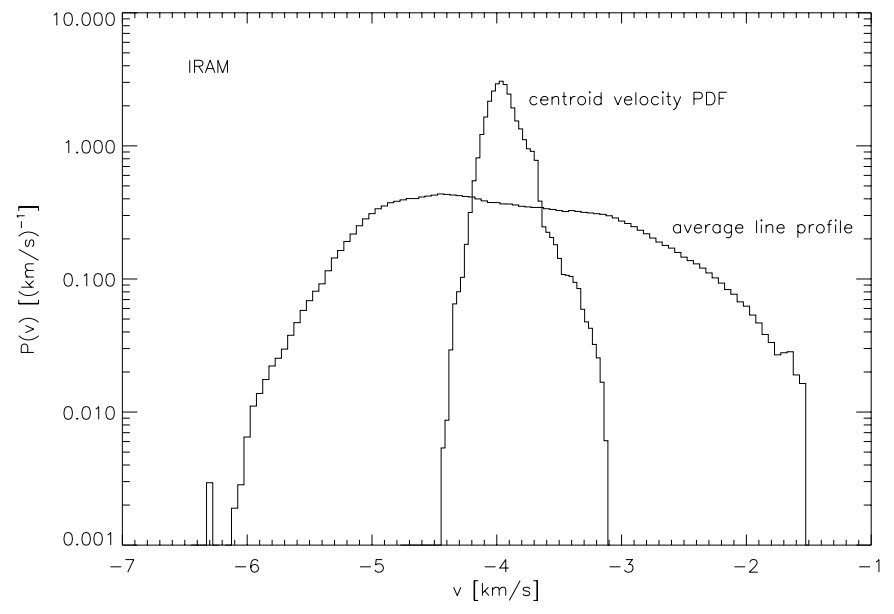

Fig. 4. Comparison of the total velocity PDF given by the average line profile and the centroid velocity PDF for the IRAM data.

where the map size is about equal to the thickness of the cloud. From the intensity maps of the clouds and Fig. 1 we estimate a thickness corresponding to about $2^{\circ}$ in angular scale. The resulting typical value for the width ratio that we should reproduce in the turbulence simulations then falls between about 1.5 and 1.6. We will see that several but not all turbulence models show such values.

We can resolve the long lasting dispute over whether to use the average line profiles or the centroid velocity PDF as a measure for the 3D velocity PDF. The answer is determined by the size scales involved in the observations. Since the velocity centroids ignore the integration along the line of sight, they provide the correct distribution when the map size is larger than or comparable to the thickness of the cloud, while for small maps, the line profiles provide the better average, because they include a larger sample from the longer line-of-sight integration.

We have to mention two caveats. First, optical depth effects can broaden lines. However, Bensch et al. (2001a) have found that the ${ }^{13} \mathrm{CO}$ and ${ }^{12} \mathrm{CO}$ Polaris data show the same spatial scaling laws, although the maps differ, suggesting that optical depth effects play only a minor role. Second, the two methods can only be equivalent for an isotropic medium, which is not guaranteed. 


\subsection{Velocity difference PDFs}

From the velocity centroid maps one can also extract information on the distribution of scales in the velocity field by considering PDFs of velocity differences between points separated by different lags (distances). This provides independent information on the structure of the velocity field. Investigation of the PDF of velocity differences as a function of spatial separation has been pursued by Miesch \& Scalo (1995), Lis et al. (1998), and Miesch et al. (1999). For a discussion of the details and the application to several molecular clouds we refer to Miesch et al. (1999).

Here we don't study the full PDF of centroid velocity differences but the variation of the first statistical moments of this PDF as a function of lag between the two points considered. Because of the symmetry of the velocity differences, all odd moments vanish. The first two non-zero moments of the velocity difference distribution are the variance and the kurtosis:

$$
\begin{aligned}
& \sigma^{2}(L)= \frac{\int_{\operatorname{map}} \mathrm{d}^{2} \boldsymbol{r} \iint_{\left|\boldsymbol{r}-\boldsymbol{r}^{\prime}\right|=L} \mathrm{~d}^{2} \boldsymbol{r}^{\prime} f(\boldsymbol{r}) f\left(\boldsymbol{r}^{\prime}\right)\left[v_{\mathrm{c}}(\boldsymbol{r})-v_{\mathrm{c}}\left(\boldsymbol{r}^{\prime}\right)\right]^{2}}{\int_{\text {map }} \mathrm{d}^{2} \boldsymbol{r} \iint_{\left|\boldsymbol{r}-\boldsymbol{r}^{\prime}\right|=L} \mathrm{~d}^{2} \boldsymbol{r}^{\prime} f(\boldsymbol{r}) f\left(\boldsymbol{r}^{\prime}\right)} \\
& K(L)=\frac{\int_{\operatorname{map}} \mathrm{d}^{2} \boldsymbol{r} \int_{\left|\boldsymbol{r}-\boldsymbol{r}^{\prime}\right|=L} \mathrm{~d}^{2} \boldsymbol{r}^{\prime} f(\boldsymbol{r}) f\left(\boldsymbol{r}^{\prime}\right)\left[v_{\mathrm{c}}(\boldsymbol{r})-v_{\mathrm{c}}\left(\boldsymbol{r}^{\prime}\right)\right]^{4}}{\sigma^{4}(L) \int_{\text {map }} \mathrm{d}^{2} \boldsymbol{r} \int_{\left|\boldsymbol{r}-\boldsymbol{r}^{\prime}\right|=L} \mathrm{~d}^{2} \boldsymbol{r}^{\prime} f(\boldsymbol{r}) f\left(\boldsymbol{r}^{\prime}\right)} .
\end{aligned}
$$

Integrations over the spatial vectors $\boldsymbol{r}$ and $\boldsymbol{r}^{\prime}$ scan the whole map. The contribution of the velocity difference between the points $\boldsymbol{r}$ and $\boldsymbol{r}^{\prime}$ is weighted by weighting factors $f$. We compared equal weighting as used by Miesch et al. (1999), weighting by the geometric mean, and by the product of the two intensities, and find little difference. In the following we use the weighting by the geometric mean, as it is a linear intensity weighting for each term, as in the case of the PDFs discussed above.

The quantity given by the variance of the two-point PDF $\sigma^{2}(L)$ in Eq. (5) as a function of the lag between the points $L$ is identical to the ordinary structure function as used e.g. by Miesch \& Bally (1994), except for the normalisation of the structure function, so we can compare the results. Miesch et al. (1999) obtained for several clouds a power law behaviour for the variance of the centroid velocity differences $\sigma^{2}(L) \propto L^{\gamma}$, with $\gamma \approx 0.85(0.33 \ldots 1.05)$ except for the largest lags, where $\sigma^{2}$ remained roughly constant. To enable a better comparison with the size-linewidth relation discussed above, we use here the standard deviation $\sigma$ instead of the variance $\sigma^{2}$. In Fig. 5 we show the resulting plot for the Polaris Flare data. We find an overall slope of 0.47 , quite close to that found for the sizelinewidth relation. The error bars are somewhat smaller but the two data sets at higher resolution show some decrease of the slope at the largest lag. This must be an artifact due to the finite map size, since it does not continue at the next larger scale. The structure functions calculated by Miesch et al. (1999) show a much stronger flattening at large lags, going to constant values for all maps. This is probably due to the artificial removal of

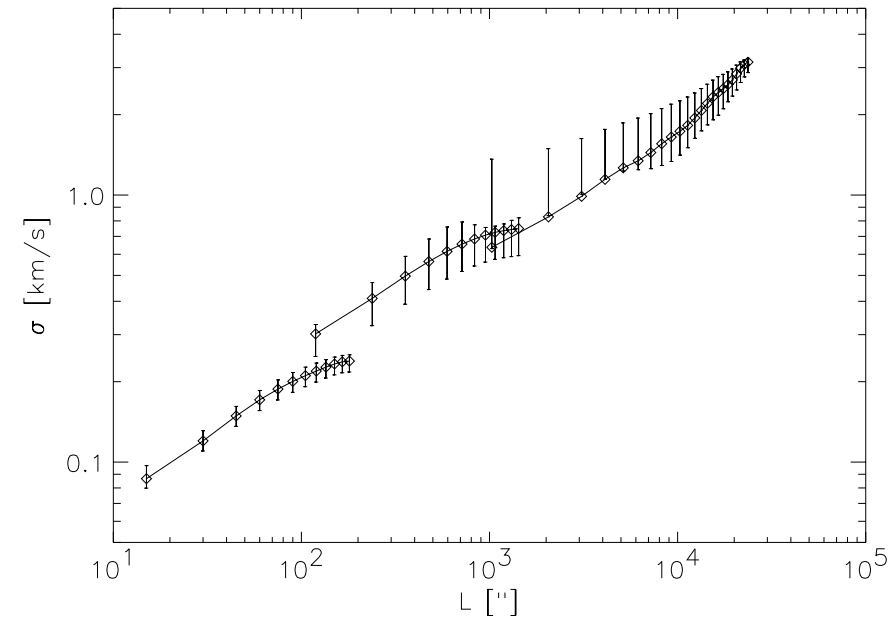

Fig. 5. The standard deviation of the velocity difference PDF as a function of lag.

velocity structure at large lags introduced by their method to subtract large scale trends.

The good agreement between the size-linewidth relation for the centroid velocities in Sect. 3.1 and the structure function discussed here seems inevitable when we consider that the variance in velocity differences on a certain scale is a kind of differential measure for the total variance within a certain radius as measured with the scanning-beam size-linewidth relation. We thus expect a similar behaviour.

The kurtosis $K(L)$ is a measure for the correlation of the internal motions. Values exceeding three at different lags indicate the strength of the correlation in velocity space at those scales. Miesch et al. (1999) found that the velocity difference PDFs in the studied clouds change from kurtosis values between about 10 and 30 at small lags to nearly Gaussian behaviour at large lags. This is also typical for incompressible turbulence (She 1991). Lis et al. (1998) found strong non-Gaussian distributions at scales associated with filaments and approximately Gaussians at larger lags.

In Fig. 6 the kurtosis of centroid velocity differences in the Polaris Flare data is plotted. In contrast to the other quantities, the different resolutions do not line up here to a single line but the kurtosis for each map drops independently to the Gaussian value at about the map size. This behaviour can be understood by considering which quantities at which scales determine the kurtosis. At the largest lag of any map the kurtosis measures mainly the shape of the centroid probability distribution of the whole map which is more or less close to Gaussian for all data sets considered here (Sect. 3.2.2). Thus we can always expect a value around 3 when the scale for the kurtosis determination approaches the map size. Contrary to the discussion provided by Miesch et al. (1999), this does not mean that there are no correlations at larger scales but that they cannot be addressed from points within the map.

At all smaller lags the kurtosis is a measure for the correlated motions on that scale relative to the overall motions seen in the map, which is scanned when computing the kurtosis and variance. In Sect. 3.3 we will see that kurtosis values above three are produced only if the maps contain some motion on 


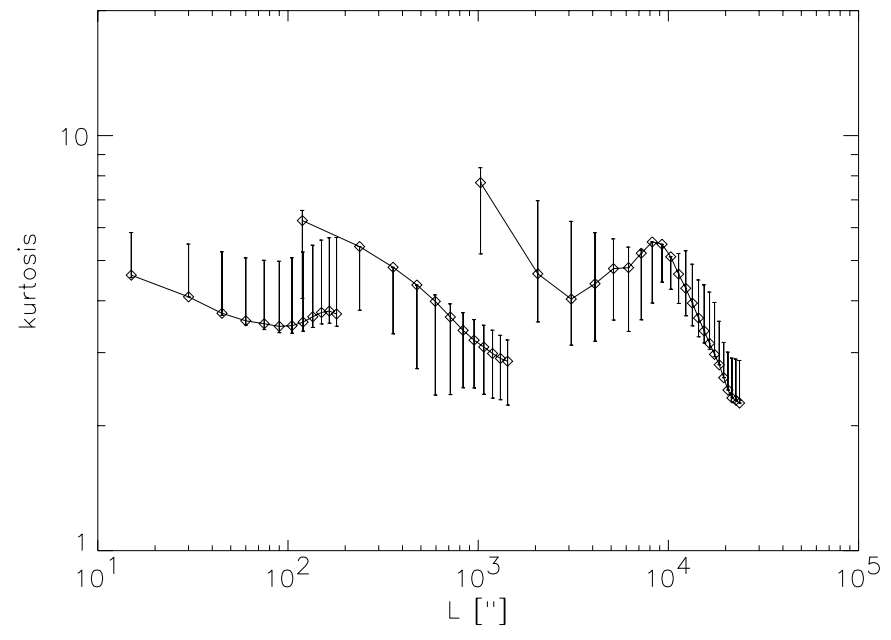

Fig. 6. The kurtosis of the centroid velocity difference PDF as a function of the lag between the points considered.

scales larger than the scale on which the kurtosis is measured. The steps in Fig. 6 are thus unavoidable when switching to another map since we always measure the correlated motions on a particular scale relative to the total motions in the map considered. A slightly sub-Gaussian behaviour at the largest scale might be produced by optical depth effects somewhat flattening the core of the distribution.

\subsection{The $\Delta$-variance}

Stutzki et al. (1998) introduced the $\Delta$-variance to measure the amount of structure present at different scales in multidimensional data sets. The $\Delta$-variance at a given scale of an $n$-dimensional data set is computed by convolving the data with an $n$-dimensional spherical down-up-down function of that scale, and measuring the remaining variance. The $\Delta$-variance analysis computes the average variance on a certain scale similar to the structure function giving the variance of the velocity differences between two distinct points separated by a certain lag. For the $\Delta$-variance, however, the variance of the filtered map is computed, instead of the average variance of all pointto-point differences corresponding to a certain lag. Thus, the $\Delta$-variance of a smooth map with a linear gradient vanishes, while the structure function discussed above detects the gradient. The advantage of the $\Delta$-variance is its better sensitivity to specific spatial scales. It provides a good separation of systematic trends, structures on certain scales, and effects like noise. Furthermore, it allows the direct computation of the equivalent Fourier spectral index. A comprehensive discussion is given by Bensch et al. (2001a). A similar method was introduced recently by Brunt (1999) to characterise the 3D velocity structure of model cubes. He used a rectangular filter function composed of adjacent cubes of different size rather than the spherically symmetric filter function used to compute the $\Delta$-variance.

Bensch et al. (2001a) applied the $\Delta$-variance analysis to the intensity maps of the Polaris Flare discussed above. We used the same method in Paper I to analyse the density structure in turbulence simulations, and compared the results to the observational data. Paper I also discussed the $\Delta$-variance for the $3 \mathrm{D}$

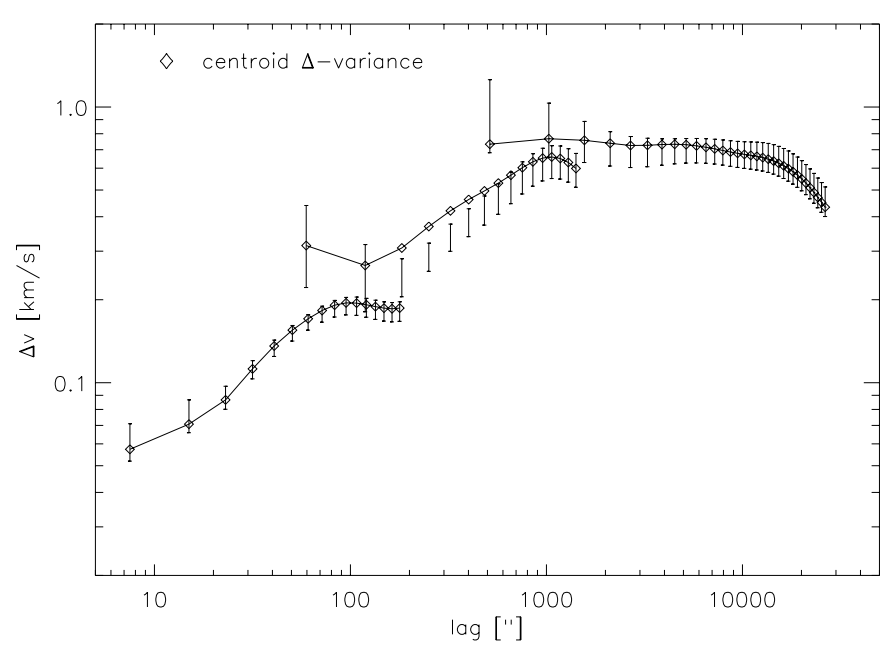

Fig. 7. The square root of the $\Delta$-variance of the velocity centroid maps of the Polaris Flare observations. In contrast to Paper I, we have chosen to take the square root, yielding the standard deviation of the filtered maps instead of the variance, in order to give a better comparison to the other linewidth related quantities.

velocity field of the simulated turbulence and compared it to the $\Delta$-variance for the $3 \mathrm{D}$ density, but did not provide any direct comparison to the observations. We found that the $\Delta$-variance of the velocity behaves similarly to that of the density in showing the characteristic scale of the driving mechanism used in the turbulence models. However, the amount of structure observed at smaller scales differs between density and velocity. The turbulence creates many thin dense regions, leading to an exponent of the $\Delta$-variance for the density of about 0.5 , whereas it creates hardly any small-scale structures in the velocity field, so that the $\Delta$-variance for the velocity drops off much more steeply, with an exponent of about 2 .

Unfortunately this method of measuring the structure of the $3 \mathrm{D}$ velocity field in the simulations has no directly equivalent approach applicable to observations, since they only provide one-dimensional velocity information projected onto the plane of the sky. There exists no simple relation between the three dimensional velocity structure and the behaviour of the projections. Therefore, we must instead apply the $\Delta$-variance analysis to observable velocity parameters like the map of centroids which can be derived from both the simulations and the observations. They can be used to judge whether a simulation reproduces observed properties. We do lose information by this procedure, of course.

Figure 7 shows the square root of the $\Delta$-variance for the Polaris Flare velocity centroid maps. The upturn at the smallest lags in each map is produced by the observational noise adding power at small scales which is only detected in the $\Delta$ variance spectrum. Bensch et al. (2001a) showed how the influence of noise can be subtracted in the $\Delta$-variance. If we apply this noise correction we find a turn-down at the smallest lags in each map. This lack of small scale variations in the different maps is due to the finite beam size of the observations blurring the structures. In the line intensity maps, the influence of the finite beam can be described by a convolution of the structure with a Gaussian beam. Bensch et al. (2001a) provided a 
formalism to correct for both the observational noise and the beam smearing, if one assumes that the internal scaling of the structure is given by a power law.

In the line centroid maps we cannot apply the same approach because the beam convolution of the channel maps that is performed by a telescope does not correspond to the convolution of the line centroid map. Estimates for random velocity fields were obtained in simulations by Miesch \& Bally (1994), but they do not provide a direct way to correct for the beam smearing in the observed data. To obtain an estimate for the uncertainty introduced by noise and beam smearing we have tested two extreme approaches. If we apply the noise correction only and disregard the smallest lags in each map, which are obviously influenced by the beam smearing, we obtain average slopes of 0.04 in the CfA map, 0.46 in the KOSMA map, and 0.68 in the IRAM map. In this case the curves do not line up perfectly. The $\Delta$-variance for one lag in a larger-scale map always falls somewhat below the $\Delta$-variance for the same lag in the smaller-scale map, indicating that the slopes are probably overestimated. If, on the other hand, we assume that the beam provided a simple convolution of the centroid map, so that we can apply Eq. (15) from Bensch et al. (2001a), we obtain slopes of $-0.05,0.36$, and 0.32 , respectively and the opposite misalignment. The true slopes should fall in between these two extreme cases.

Over most scales covered by the CfA map, the slope flattens to zero, in contrast to the behaviour in the intensity maps. This virtual lack of large-scale variations does not reflect the real structure but appears to be due to the missing weighting in the $\Delta$-variance analysis. Here, all points are counted equally, even those with intensities well below the noise limit. Thus, the $\Delta$ variance analysis necessarily fails in cases where the maps are only sparsely filled by emission. Less than one third of the CfA map shows emission above the noise limit so that we cannot expect any significant results at scales above half a degree.

For the maps at smaller scales without large "empty" regions, we can expect significant $\Delta$-variance spectra but we must admit that without a good theory for the influence of the beam convolution on line centroids we can hardly separate the effects of the beam smearing from the velocity scaling in maps which are not large compared to the beam size. Thus large error bars remain for the measured velocity scaling in the Polaris Flare preventing any definite conclusion on the scale dependence of the slope in the velocity structure.

In contrast the size-linewidth relation and the structure function do not show clear signatures of noise and beam smearing in Figs. 1 and 5. To test their sensitivity we have applied them to data smoothed with a Gaussian filter tuned to ensure that the corresponding $\Delta$-variance shows the same behaviour as the $\Delta$-variance where the noise was removed following Bensch et al. (2001a). Only small changes relative to the original data are seen. The size-linewidth relation and the structure function of the velocity centroids exhibit a weak steepening of the slope by about 0.04 , while the velocity PDFs and the kurtosis show no clear differences. The comparison of the results from the original maps and the smoothed data shows that these other methods are not particularly sensitive to uncorrelated noise or beam smearing at small scales.

\subsection{Comparison of the methods}

We can classify all of the methods we have described in terms of the velocity information setup in a two-dimensional map, the filtering function used, and the weighting of the data in the map.

Most analyses were restricted to the velocity centroids, which are effectively the first moment of the local velocity profile. The size-linewidth relation adds the local variance, i.e. the second velocity moment, and the study of the PDFs also uses the kurtosis. However, with sufficiently high signal-to-noise, higher moments may provide valuable additional information.

The variance is strongly dominated by the depth of the observed cloud, so that it contains information lost when considering the velocity centroids only. We have seen that, for maps where the line profiles sample the cloud deeply in comparison to the map size, the integrated line profile is a better measure for the true velocity distribution function than the PDF of the velocity centroids.

We have applied three different kinds of filters: the scanning-beam size-linewidth relation effectively convolves the map with a positive Gaussian filter; the $\Delta$-variance analysis uses a spherically symmetric up-down filter; and the structure function uses a filter consisting of a positive and a negative spike separated by a certain distance. In the latter case, spherical symmetry is provided by the superposition of the resulting variance values for different directions of the filter axis. The structure function is sensitive to large-scale gradients and can detect certain geometric structures, but because of the strong localisation of the filter in the spatial domain, it is unfortunately sensitive to a broad spectrum of spatial frequencies in the Fourier domain. In the statistical analysis of velocity fluctuations, it is therefore at a disadvantage in the detection of characteristic scales and frequencies compared to the $\Delta$-variance analysis. Similar conclusions were obtained by Houlahan \& Scalo (1990). This may explain why we detect the scale of noise only by means of the $\Delta$-variance, whereas its influence is hidden in the full spectra of the structure function and the size-linewidth relation.

The $\Delta$-variance analysis, on the other hand, does not yet take into account different weights for the information in different regions of an observed map, so that it fails for maps with large regions dominated by noise. The weighting of the velocity centroid information by the intensity, as is done automatically in the size-linewidth relation, reduces the uncertainty due to observational noise when computing the centroid probability distribution or structure function.

\subsection{Other approaches}

With sufficiently high signal-to-noise ratio, it is possible to extend the methods discussed here. Overviews of the different existing methods have been provided recently by VázquezSemadeni (2000) and Ossenkopf et al. (2000). First, one can apply the basically two-dimensional methods to higher moments of the line profiles, providing new information especially on the intermittency in velocity space. Alternatively, the velocity channel maps can be analysed as demonstrated with the 
$\Delta$-variance analysis by Ossenkopf et al. (1998). Another method is to compare full spectra, using the spectral correlation function (Rosolowsky et al. 1999) and extending this method to consider all spatial variations.

Tauber (1996) discussed the smoothness of line profiles as a measure for the size and number of coherent units contributing to the profiles. Applying a rough approximation to this analysis, Falgarone et al. (1998) conclude that the size of cells in the Polaris Flare observations must be as low as 200 AU.

When looking for characteristic global features in the density-velocity structure, the principal component analysis introduced by Heyer \& Schloerb (1997) is probably the most significant tool. It identifies the main components in the positionvelocity space in terms of eigenvectors and eigenimages. Although the principal component analysis represents a reliable method to find the dominant structures even in complicated images, the significance of the higher-order moments still has to be determined.

\section{Turbulence models}

\subsection{Simulations}

We use simulations of uniform decaying or driven turbulence with and without magnetic fields described by Mac Low et al. (1998) in the decaying case and by Mac Low (1999) in the driven case. These simulations were performed with the astrophysical MHD code ZEUS-3D ${ }^{2}$ (Clarke 1994). This is a 3D version of the code described by Stone \& Norman (1992a,b) using second-order advection (van Leer 1977), that evolves magnetic fields using constrained transport (Evans \& Hawley 1988), modified by upwinding along shear Alfvén characteristics (Hawley \& Stone 1995). The code uses a von Neumann artificial viscosity to spread shocks out to thicknesses of three or four zones in order to prevent numerical instability, but contains no other explicit dissipation or resistivity. Structures with sizes close to the grid resolution are subject to the usual numerical dissipation, however. In Paper I we discussed the effects of limited numerical resolution, which leads to numerical viscosity, and noted that resolution studies could be used to determine which properties were well resolved.

The simulations used here were performed on a $3 \mathrm{D}$, uniform, Cartesian grid with side $L=2$ and periodic boundary conditions in every direction, using an isothermal equation of state. To deal with velocities comparable to those in the observations we have assumed here a cloud temperature of $10 \mathrm{~K}$ corresponding to a translation of the dimensionless sound speed in the simulations to a physical sound speed of $0.2 \mathrm{~km} \mathrm{~s}^{-1}$ in the data analysis. The initial density and, in relevant cases, magnetic field are both initialised uniformly on the grid, with the initial density $\rho_{0}=1$ and the initial field parallel to the $z$-axis. The turbulent flow is initialised with velocity perturbations drawn from a Gaussian random field determined by its power

\footnotetext{
2 Available from the Laboratory for Computational Astrophysics of the National Center for Supercomputing Applications, http://zeus.ncsa. uiuc.edu/lca_home_page.html
}

distribution in Fourier space, as described by Mac Low et al. (1998). For decaying models we use a flat spectrum with power in the range $k_{\mathrm{d}}=1-8$, where the dimensionless wavenumber $k_{\mathrm{d}}=L / \lambda_{\mathrm{d}}$ counts the number of driving wavelengths $\lambda_{\mathrm{d}}$ in the box. For our driven models, we use a spectrum consisting of a narrow band of wave numbers around some value $k_{\mathrm{d}}$, and driven with a fixed pattern at constant kinetic energy input rate, as described by Mac Low (1999).

We have tested the influence of numerical viscosity by running the simulations with the same physical parameters on grids of $64^{3}, 128^{3}$ or $256^{3}$ zones. Higher resolution grids have numerical viscosity acting at smaller scales, so changing the resolution shows the effects of numerical viscosity on our results. The influence of numerical resolution on the simulation results is discussed below separately for each for the statistical measures.

\subsection{Simulated observations}

To compare our simulations with observations we must synthesise observational maps from the simulated density and velocity fields. We assume that the cubes are optically thin for this first study so that direct integration along lines of sight through the cube neglecting optical depth effects yields line profiles. This appears to be a reasonable assumption for comparison with the low column density clouds in the Polaris flare observed in ${ }^{13} \mathrm{CO}$, but is a worse assumption for higher column density clouds or more optically thick species.

We also neglect the periodic nature of the simulations, effectively observing the simulation cubes as isolated structures in a vacuum. This second assumption must be taken into account in analyses affected by the path-length through the cloud, such as comparisons of the velocity PDF measured from the average line profile vs. the centroid velocity distribution.

\subsection{Statistical fluctuations}

To get a feeling for the significance of the structural properties indicated by the different measures relative to the statistical variations in the turbulence, which may build up from isotropic initial perturbations during the turbulence evolution, we can compare different directions within the same model cube.

Figure 8 shows the $\Delta$-variances and structure functions for the three centroid velocity maps along the three axes in a decaying hydrodynamic model. The $\Delta$-variances show variations of $40 \%$ in value at the largest scales, where the exact modal structure is still different in the three directions. The structure function shows smaller variations corresponding to its lower sensitivity to changes at particular spatial scales. The variation of the slope in the different directions falls below 0.1 . The equivalent plot for the size-linewidth relation is similar to that for the structure function. The variations in the kurtosis, on the other hand, are more like those seen in the $\Delta$ variance. Here, we sometimes find small dips and rises distorting the monotonic decay to values slightly below 3 at the largest scales. In the total and the centroid velocity PDFs we find substantial variations in the core and the central position of the 

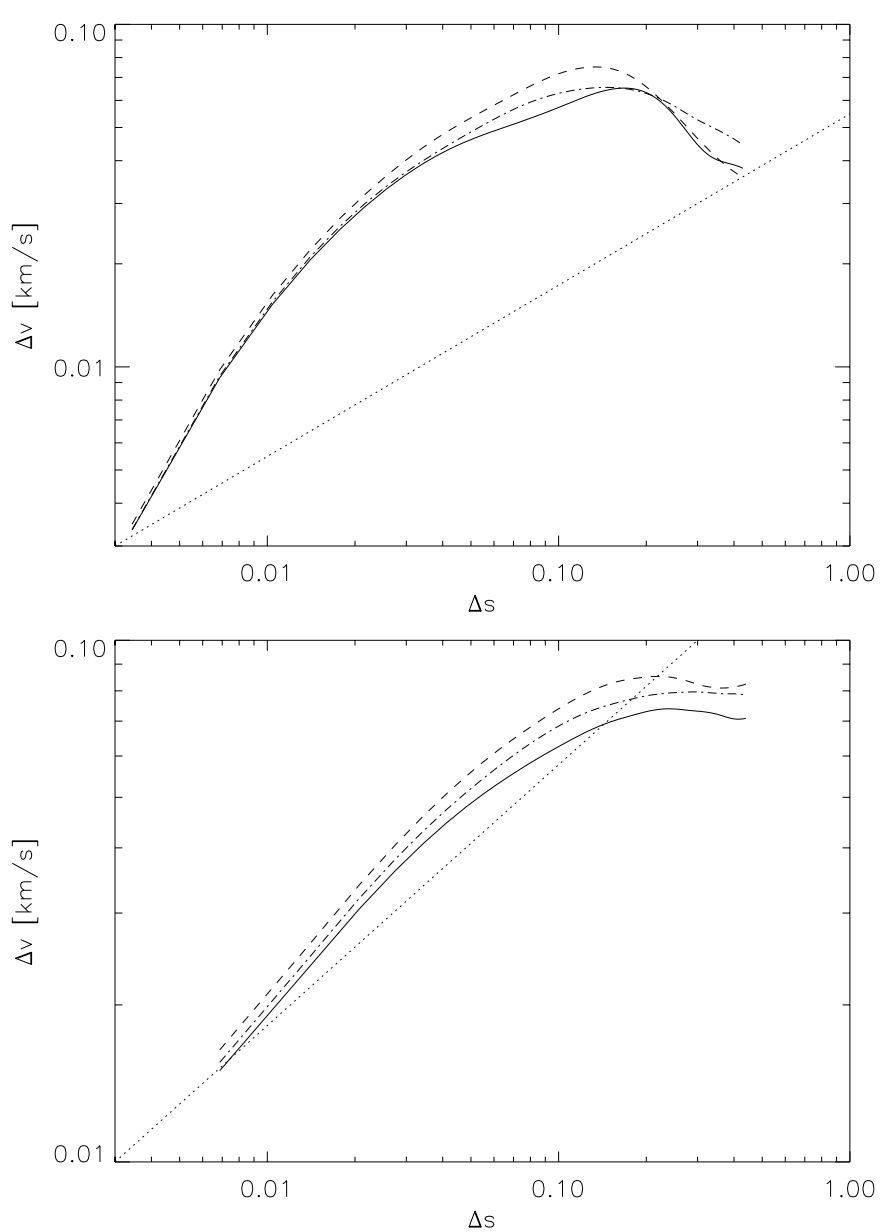

Fig. 8. $\Delta$-variances (upper panel) and structure functions (lower panel) in the centroid velocity maps obtained for a decaying hydrodynamic model (model D from Mac Low et al. 1998) in the three possible directions of projection. The dotted line indicates a slope of 0.5 for comparison. The variation between the different directions is similar for the other models. In the $\Delta$-variance plots we always give the standard deviation (the square root of the $\Delta$-variance) for a better comparison to the other velocity variations.

distributions corresponding to the different largest velocity modes but no changes in the wing behaviour.

\section{Statistical description of simulations and comparison to observations}

\subsection{Size-linewidth relation}

We begin by considering the results of applying the sizelinewidth analysis described in Sect. 3.1 to the models. In Fig. 9 we show the size-linewidth relation for the velocity centroids in three models of hydrodynamic driven turbulence that differ only in the scale that they are driven. The driving wavelengths are $1 / 2,1 / 4$, and $1 / 8$, respectively.

We find power-law behaviour through most of the regime only for the model driven at the largest available scales. Models driven with smaller characteristic scales show a flattening of the relation at lags above the driving scale. A slight flattening at the largest lags is also visible in the observational data. This appears to be an indication of a turbulence driving scale close

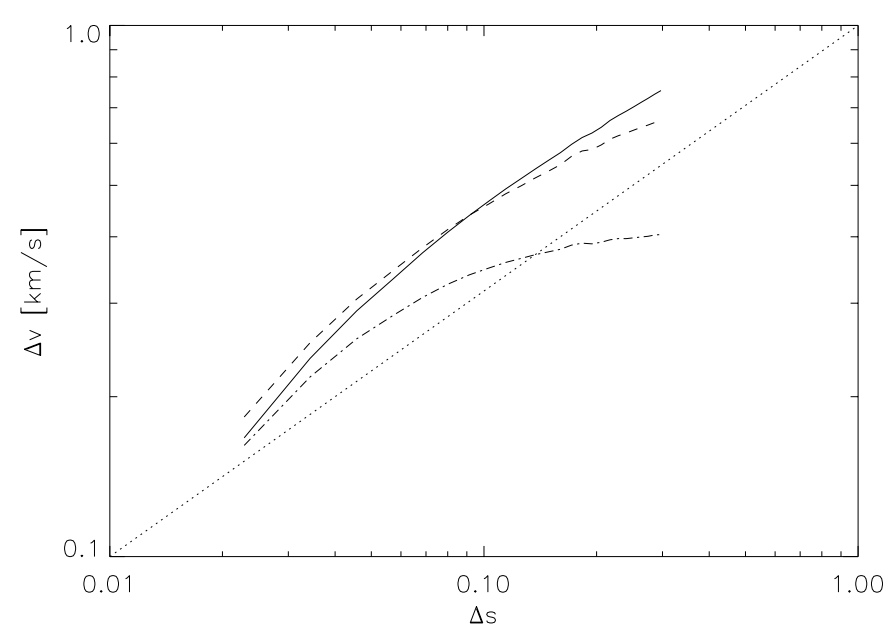

Fig. 9. Size-linewidth relation for the velocity centroids of a hydrodynamic simulation driven at wavenumbers of $k_{\mathrm{d}}=2$ (solid), $k_{\mathrm{d}}=4$ (dashed), and $k_{\mathrm{d}}=8$ (dot-dashed). These are models HE2, HE4, and HE8 from Mac Low (1999). The dotted line shows a power-law with slope 0.5 .

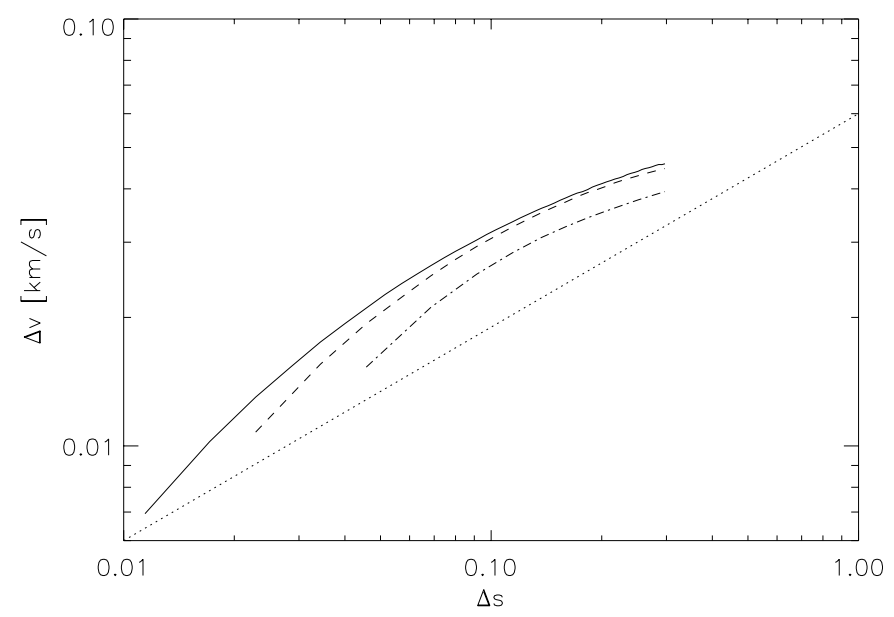

Fig. 10. Size-linewidth relation for the velocity centroids in a hydrodynamic simulation of decaying turbulence at time $t=0.75 t_{\text {cross }}\left(t_{\text {cross }}\right.$ is the initial crossing time of the region), computed at resolutions of $64^{3}$ (dot-dashed), $128^{3}$ (dashed), and $256^{3}$ (solid). These are models B, C, and D from Mac Low et al. (1998).

to the size of the molecular cloud. A drop off in velocity dispersion is seen at small lags in all of the models. This can be explained straightforwardly as an effect of numerical viscosity. In Fig. 10 we show a comparison of three models of decaying turbulence that are statistically identical, but were computed at resolutions of $64^{3}, 128^{3}$, and $256^{3}$ zones. Increasing resolution results in decreasing numerical viscosity, so Fig. 10 demonstrates explicitly the effect of changing the numerical viscosity. As can be seen, the slope does not change at large lags, and the higher resolution models agree within a few percent on the magnitude of the velocity dispersion. At small lags, on the other hand, the velocity dispersion falls off. This occurs in all models at roughly the same number of grid zones, and hence at larger physical scale in the lower resolution models. This part of the spectrum thus reflects the effect of numerical diffusion eliminating some of the small-scale structure. The same 

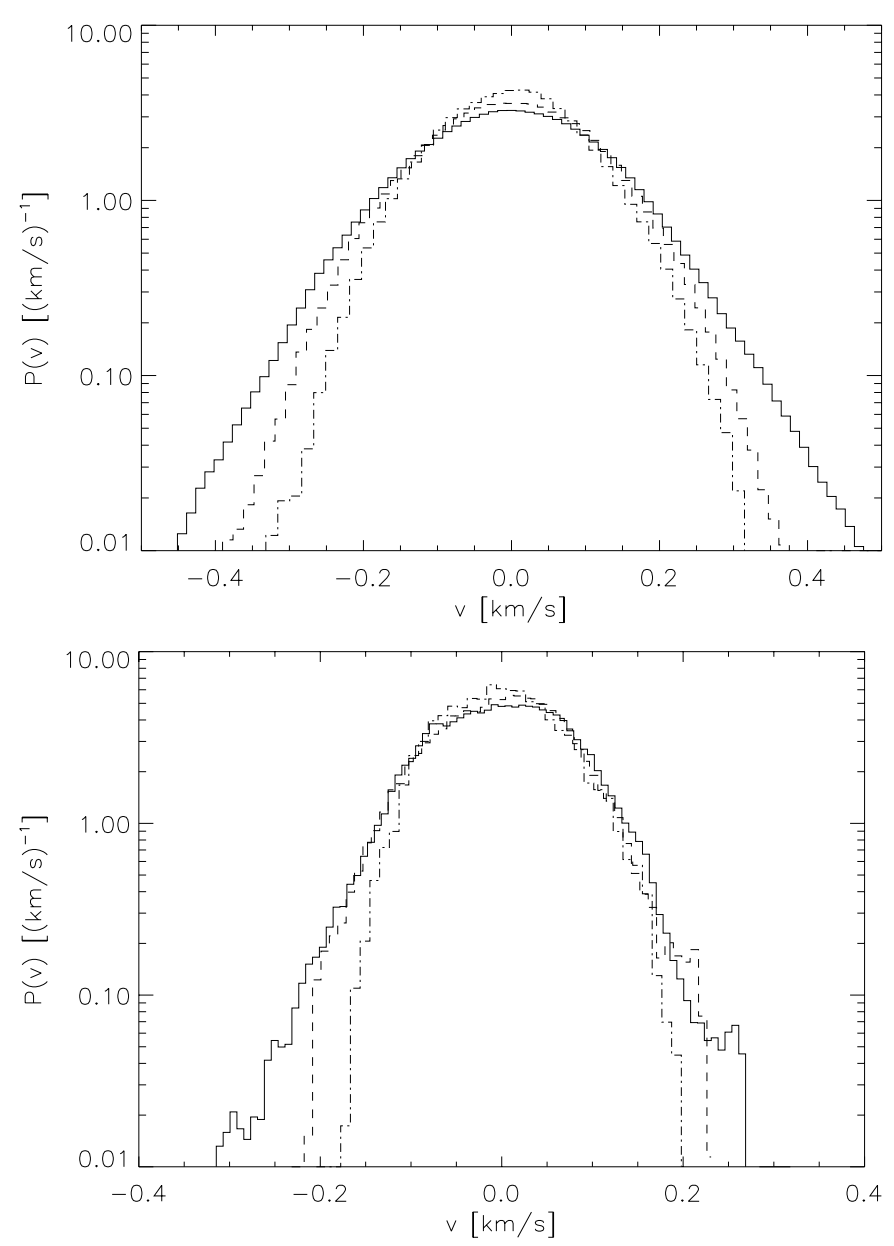

Fig. 11. a) $3 \mathrm{D}$ and b) centroid velocity PDFs in a model of strongly magnetised decaying turbulence with initial Mach number $M=5$ and Alfvén number $A=1$ after $1.5 t_{\text {cross }}$ at a resolution of $256^{3}$ (solid), $128^{3}$ (dashed), and $64^{3}$ (dashed-dotted). (Models Q, P, N from Mac Low et al. 1998).

drop-off occurs in each model (see Fig. 9), so the behaviour at the smallest scales should be viewed with caution. We note, however, that a physical diffusion such as ambipolar diffusion (Zweibel \& Josafatsson 1983; Klessen et al. 2000) is expected to produce similar behaviour at the diffusion scale.

Magnetic fields do not appear to modify the size-linewidth relation, although they can make order unity differences in the magnitude of the velocity dispersion and produce significant anisotropy, as described below in Sect. 5.4.

We can conclude that the observed power-law behaviour of the size-linewidth relation is reasonably explained by either hydrodynamic or magnetised turbulence driven at scales comparable to the largest observed scales.

\subsection{Velocity probability distribution function}

\subsubsection{Centroid velocity PDFs}

We begin our study of velocity PDFs in the models by examining the effect of numerical resolution on the centroid velocity PDFs. In Fig. 11 the 3D PDFs are compared to the centroid PDFs for a model of decaying MHD turbulence at different

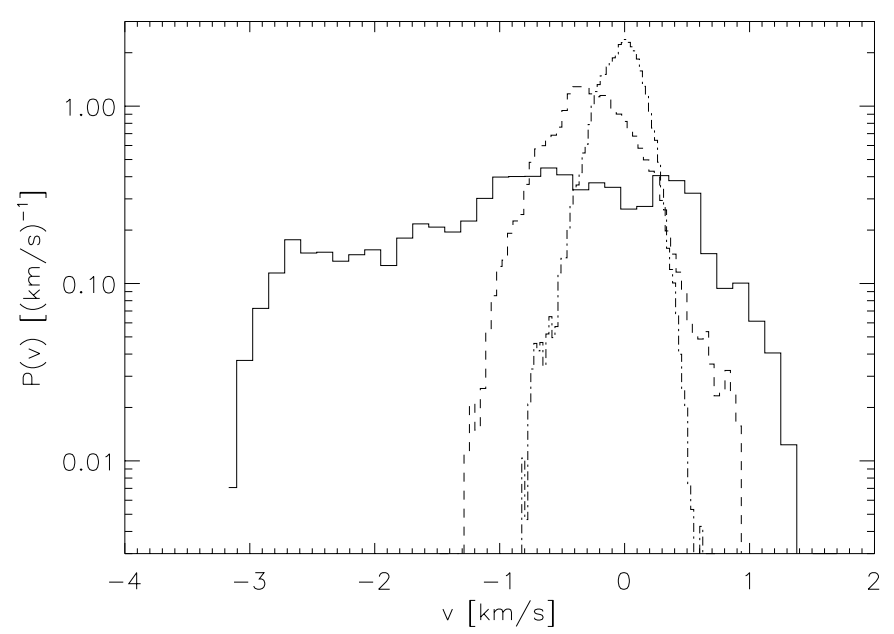

Fig. 12. Centroid velocity PDFs from three models driven with the same strength at wavenumbers of $k_{\mathrm{d}}=2$ (solid), $k_{\mathrm{d}}=4$ (dashed), and $k_{\mathrm{d}}=8$ (dash-dotted) (models HC2, HC4, and HC8 from Mac Low 1999).

resolutions. All PDFs are well represented by Gaussians. The width of the PDF drops at smaller resolution. This can be attributed to the stronger influence of the numerical viscosity in the lower resolution cubes damping the turbulence faster. This agrees with the measurements of resolution effects on kinetic energy described in Mac Low et al. (1998), in which the magnitude of the kinetic energy increased with resolution, although the decay rate was constant. An additional effect is seen in the centroid PDFs. At low resolutions the sampling of the wings of the Gaussian is insufficient, so that the distribution appears too narrow. The kurtosis values of these PDFs are 2.6, 2.8, and 2.9 with growing resolution. Hence, sub-Gaussian kurtosis values can at least partially be explained by small map sizes.

In Table 2 we give the PDF moments for most of the models discussed, covering a wide range of different physical properties. The first several columns describe the model input parameters, and the remaining columns contain the parameters of the PDFs obtained.

We first consider the effect of varying the driving wavenumber, holding the energy input constant. Figure 12 and the corresponding values in Table 2 show that driving at smaller wavenumbers (longer wavelengths) produces broader PDFs, because such models have lower dissipation rates (Mac Low 1999), and thus higher rms velocities. More interestingly, we find that driving at the largest scale in the model $k_{\mathrm{d}}=2$ produces a centroid velocity PDF with apparently non-Gaussian shape, as reflected in the kurtosis value of 2.4 given in Table 2. A similar result was found by Klessen (2000), who argued that it is most likely due to cosmic variance. That is, an insufficient number of modes are sampled at these long driving wavelengths to fully describe a Gaussian field, so the PDF appears to have a distorted shape. Depending on the random numbers used to initialise the largest modes, both Gaussian and non-Gaussian kurtosis values are then possible.

To demonstrate the effect of magnetic fields, Fig. 13 shows the centroid velocity PDFs from a model of decaying magnetised turbulence. It clearly indicates an anisotropic decay, with 
Table 2. Parameters of the centroid velocity PDFs and the total velocity PDFs for the model cubes.

\begin{tabular}{|c|c|c|c|c|c|c|c|c|c|c|c|}
\hline $\operatorname{model}^{a}$ & $L^{b}$ & $k_{\mathrm{d}}^{c}$ & $N^{d}$ & $M^{e}$ & $\frac{v_{\mathrm{A}}}{c_{\mathrm{s}}} f$ & $t^{g}$ & $\frac{\sigma^{2}}{c_{\mathrm{s}}^{2}}(\operatorname{cen})^{h}$ & $\frac{\sigma^{2}}{c_{\mathrm{s}}^{2}}(\text { cube })^{h}$ & $\frac{\sigma^{2}(\text { cube })}{\sigma^{2}(\text { cen })}$ & $K(\text { cen })^{j}$ & $K(\text { cube })^{j}$ \\
\hline HA8 & 0.1 & $7-8$ & 128 & 1.9 & 0 & 1.0 & 3.6 & 10 & 2.8 & 3.0 & 2.9 \\
\hline $\mathrm{HC} 2$ & 1 & $1-2$ & 128 & 7.4 & 0 & 1.0 & 50 & 55 & 1.1 & 2.4 & 2.5 \\
\hline $\mathrm{HC} 4$ & 1 & $3-4$ & 128 & 5.3 & 0 & 1.0 & 17 & 31 & 1.8 & 3.2 & 2.7 \\
\hline $\mathrm{HC} 8$ & 1 & $7-8$ & 128 & 4.1 & 0 & 1.0 & 9.4 & 22 & 2.3 & 3.6 & 3.0 \\
\hline HE2 & 10 & $1-2$ & 128 & 15 & 0 & 0.98 & 49 & 76 & 1.6 & 3.0 & 2.7 \\
\hline HE4 & 10 & $3-4$ & 128 & 12 & 0 & 0.88 & 38 & 67 & 1.8 & 2.9 & 3.0 \\
\hline HE8 & 10 & $7-8$ & 128 & 8.7 & 0 & 1.0 & 21 & 47 & 2.2 & 3.9 & 3.0 \\
\hline MA4X: $v_{\perp}$ & 0.1 & $3-4$ & 128 & 2.7 & 10 & 0.3 & 7.0 & 14 & 2.0 & 2.6 & 2.8 \\
\hline$v_{\|}$ & 0.1 & $3-4$ & 128 & 2.7 & 10 & 0.3 & 10 & 16 & 1.6 & 3.4 & 3.3 \\
\hline MC4X: $v_{\perp}$ & 1 & $3-4$ & 128 & 5.3 & 10 & 0.1 & 15 & 26 & 1.7 & 3.8 & 3.6 \\
\hline$v_{\|}$ & 1 & $3-4$ & 128 & 5.3 & 10 & 0.1 & 18 & 29 & 1.6 & 3.3 & 3.3 \\
\hline MC45: $v_{\perp}$ & 1 & $3-4$ & 128 & 4.8 & 5 & 0.2 & 13 & 23 & 1.8 & 4.1 & 3.4 \\
\hline$v_{\|}$ & 1 & $3-4$ & 128 & 4.8 & 5 & 0.2 & 16 & 29 & 1.8 & 3.1 & 3.0 \\
\hline MC41: $v_{\perp}$ & 1 & $3-4$ & 128 & 4.7 & 1 & 0.5 & 15 & 28 & 1.9 & 3.0 & 2.8 \\
\hline$v_{\|}$ & 1 & $3-4$ & 128 & 4.7 & 1 & 0.5 & 13 & 24 & 1.8 & 3.1 & 3.0 \\
\hline MC85: $v_{\perp}$ & 1 & $7-8$ & 128 & 3.4 & 5 & 0.075 & 6.3 & 15 & 2.4 & 4.1 & 3.8 \\
\hline$v_{\|}$ & 1 & $7-8$ & 128 & 3.4 & 5 & 0.075 & 9.0 & 21 & 2.3 & 3.2 & 3.5 \\
\hline $\mathrm{MC} 81: v_{\perp}$ & 1 & $7-8$ & 128 & 3.5 & 1 & 0.23 & 6.6 & 19 & 2.9 & 3.3 & 3.1 \\
\hline$v_{\|}$ & 1 & $7-8$ & 128 & 3.5 & 1 & 0.23 & 8.5 & 20 & 2.4 & 3.3 & 3.2 \\
\hline $\operatorname{ME} 21^{k}: v_{\perp}$ & 10 & $1-2$ & 128 & 14 & 1 & 0.5 & 47 & 61 & 1.3 & 2.8 & 2.9 \\
\hline$v_{\|}$ & 10 & $1-2$ & 128 & 14 & 1 & 0.5 & 83 & 97 & 1.2 & 3.3 & 3.2 \\
\hline \multirow[t]{2}{*}{ B } & 0 & $1-8$ & 64 & 5 & 0 & 0.1 & 2.7 & 7.0 & 2.6 & 2.6 & 2.9 \\
\hline & 0 & $1-8$ & 64 & 5 & 0 & 0.3 & 1.9 & 4.0 & 2.1 & 2.8 & 2.9 \\
\hline \multirow[t]{3}{*}{$\mathrm{C}$} & 0 & $1-8$ & 128 & 5 & 0 & 0.1 & 2.8 & 7.7 & 2.8 & 3.0 & 3.1 \\
\hline & 0 & $1-8$ & 128 & 5 & 0 & 0.3 & 1.7 & 4.4 & 2.6 & 3.2 & 2.9 \\
\hline & 0 & $1-8$ & 128 & 5 & 0 & 0.5 & 1.3 & 3.5 & 2.7 & 2.7 & 2.7 \\
\hline \multirow[t]{3}{*}{ D } & 0 & $1-8$ & 256 & 5 & 0 & 0.1 & 2.9 & 8.4 & 2.9 & 3.0 & 2.9 \\
\hline & 0 & $1-8$ & 256 & 5 & 0 & 0.3 & 1.9 & 5.0 & 2.6 & 2.9 & 2.9 \\
\hline & 0 & $1-8$ & 256 & 5 & 0 & 0.5 & 1.4 & 3.8 & 2.7 & 2.4 & 2.9 \\
\hline \multirow[t]{3}{*}{$\mathrm{U}^{\mathrm{k}}$} & 0 & $1-8$ & 256 & 50 & 0 & 0.1 & 9.1 & 15 & 1.6 & 2.3 & 2.9 \\
\hline & 0 & $1-8$ & 256 & 50 & 0 & 0.3 & 6.1 & 8.9 & 1.5 & 2.1 & 2.6 \\
\hline & 0 & $1-8$ & 256 & 50 & 0 & 0.5 & 5.0 & 7.0 & 1.4 & 2.1 & 2.5 \\
\hline \multirow[t]{3}{*}{ Q: $v_{\perp}$} & 0 & $1-8$ & 256 & 5 & 5 & 0.1 & 3.6 & 11 & 3.1 & 3.2 & 3.3 \\
\hline & 0 & $1-8$ & 256 & 5 & 5 & 0.3 & 2.4 & 6.4 & 2.7 & 2.9 & 3.3 \\
\hline & 0 & $1-8$ & 256 & 5 & 5 & 0.5 & 2.1 & 4.9 & 2.3 & 3.0 & 3.2 \\
\hline \multirow[t]{3}{*}{$\mathrm{Q}: v_{\|}$} & 0 & $1-8$ & 256 & 5 & 5 & 0.1 & 4.7 & 9.5 & 2.0 & 2.9 & 3.0 \\
\hline & 0 & $1-8$ & 256 & 5 & 5 & 0.3 & 3.9 & 6.3 & 1.6 & 3.0 & 3.4 \\
\hline & 0 & $1-8$ & 256 & 5 & 5 & 0.5 & 3.4 & 4.9 & 1.4 & 3.1 & 3.3 \\
\hline
\end{tabular}

${ }^{a}$ Driven models (H \& M series) from Mac Low (1999); decaying models (single letters) from Mac Low et al. (1998).

${ }^{b}$ Mechanical driving luminosity in arbitrary units (see Sect. 2.3 of Mac Low 1999, for all unit conversions).

${ }^{c}$ Driving wavenumber.

${ }^{d}$ Number of zones in each dimension.

${ }^{e}$ rms Mach number: initial value for decaying models, equilibrium value for driven models.

${ }^{f}$ Ratio of Alfvén velocity to sound speed.

${ }^{g}$ Time at which values are measured, in sound-crossing times.

${ }^{h}$ Variance of distribution for line centroid velocities and full cube.

${ }^{j}$ Kurtosis of distribution for line centroid velocities and full cube.

${ }^{k}$ Unpublished model. 

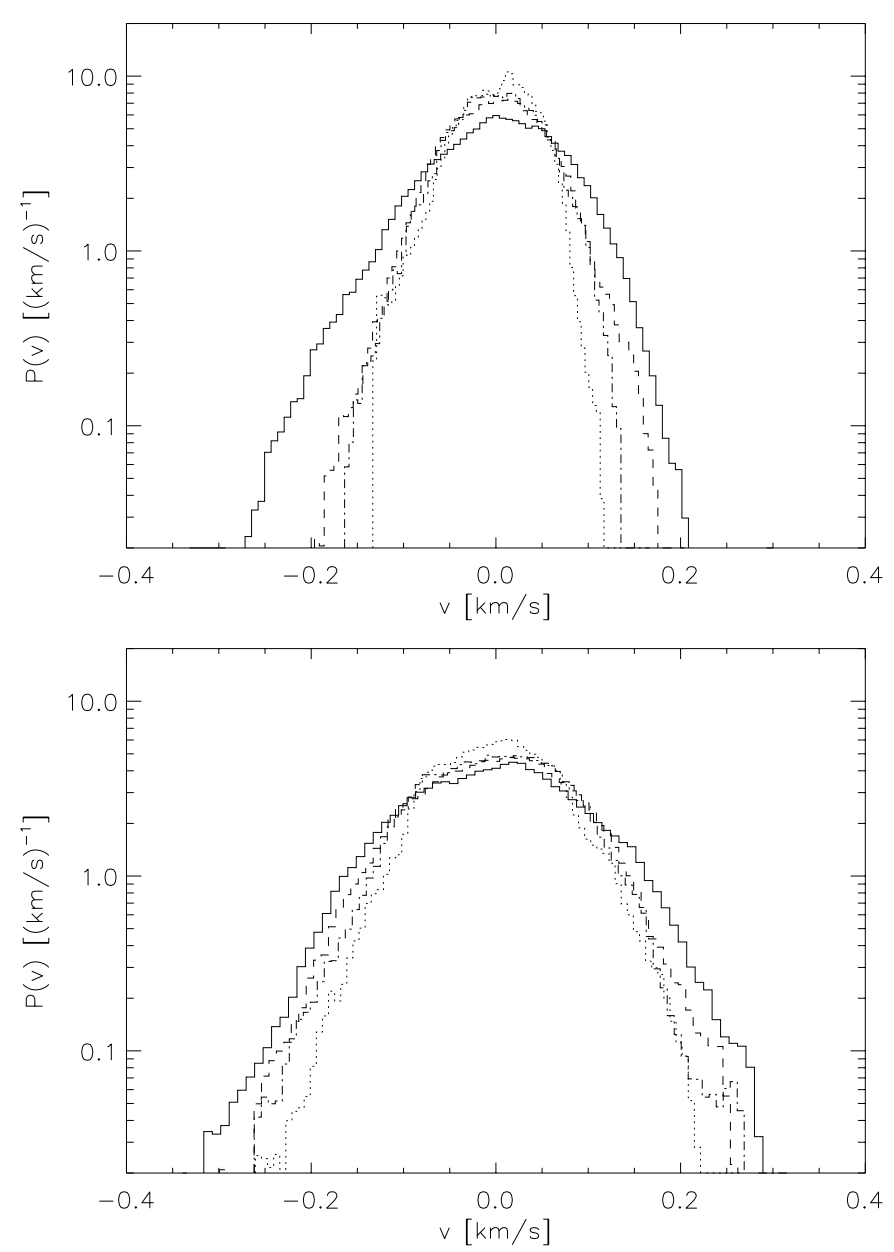

Fig. 13. Centroid velocity PDFs from a model of decaying, magnetised turbulence (model Q from Mac Low et al. 1998) at times in units of the initial crossing time $t_{\text {cross }}$ of 0.5 (solid), 0.75 (dashed), 1.5 (dash-dot), and 2.5 (dotted) observed a) perpendicular to the field and b) parallel to the field.

velocity components perpendicular to the magnetic field decaying substantially more quickly than velocities parallel to the field. In both cases, though, the PDFs remain Gaussian even at late times. These conclusions are quantitatively supported by Table 2.

We also examined magnetised driven turbulence. The fields do slightly shift the peak, but, as shown in Table 2, they still produce only marginally non-Gaussian centroid velocity PDFs in cases where the hydrodynamic model is Gaussian, contrary to the speculation of Klessen (2000) that magnetic fields might be an important alternative cause of non-Gaussian PDFs. The PDFs observed parallel to the field are roughly $20 \%$ wider than the perpendicular observations as seen in Table 2 .

Klessen (2000) showed that driving from large scales can produce non-Gaussian PDFs, but worried that every additional piece of physics also appeared likely to produce non-Gaussian PDFs, allowing no conclusions to be drawn from their occurrence. We have, however, demonstrated that neither magnetic fields nor the vorticity introduced by shock interactions in driven or decaying turbulence produce strongly non-Gaussian PDFs.

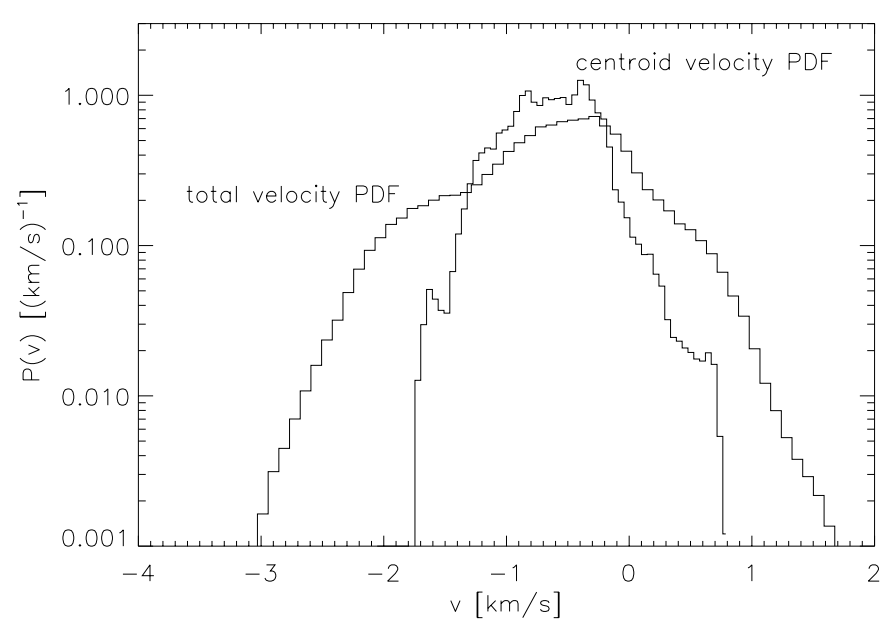

Fig. 14. Comparison of the total velocity PDF and the centroid velocity PDF for a hydrodynamic model driven with one tenth of the energy of the example above (model HC2 from Mac Low 1999).

Therefore, the non-Gaussian PDFs observed in the Polaris Flare (Sect. 3.2) must have a different explanation. Another candidate for producing non-Gaussian PDFs is self-gravity, but the lack of star-forming activity in the Polaris Flare suggests that self-gravitation does not play a dominant role there. This suggests that the non-Gaussian PDFs observed there are indeed due to the cosmic variance introduced by driving from the largest scales of the region. The driving scale may actually be even larger than identified here because we have not used any information from the atomic gas at larger scales.

\subsubsection{Average line profiles}

Using the turbulence simulations we can now revisit the question from Sect. 3.2.2 of how best to measure the actual 3D velocity PDF from observations. In Fig. 14 we compare the PDF of the whole velocity distribution that would be measured as the average line profile in an optically thin LTE medium to the PDF of the centroid velocities for a hydrodynamic model driven at $k_{\mathrm{d}}=2$. This plot may be compared with Fig. 4, showing the centroid velocity distribution and the average line profile measured for the IRAM map in the Polaris Flare.

In both cases we find similar distorted Gaussian distributions, with the centroid velocity distribution narrower than the full velocity distribution. Table 2 contains the ratio between the widths of the two distributions for all models. The model ratios show substantial variation around a typical value of about two. The strongest systematic variation appears to be with initial or driven rms Mach number $M_{0}$, with higher $M_{0}$ giving lower ratios, down to as low as 1.1 for the most strongly driven model HE2. Observing parallel to the field lines leads to somewhat lower ratios than perpendicular, presumably due to the higher velocity variance seen along parallel lines of sight. However, the ratio also decreases during the decay of turbulence for unclear reasons.

The ratio between depth and width is fixed to unity in the model. From the combination of the three Polaris Flare maps 


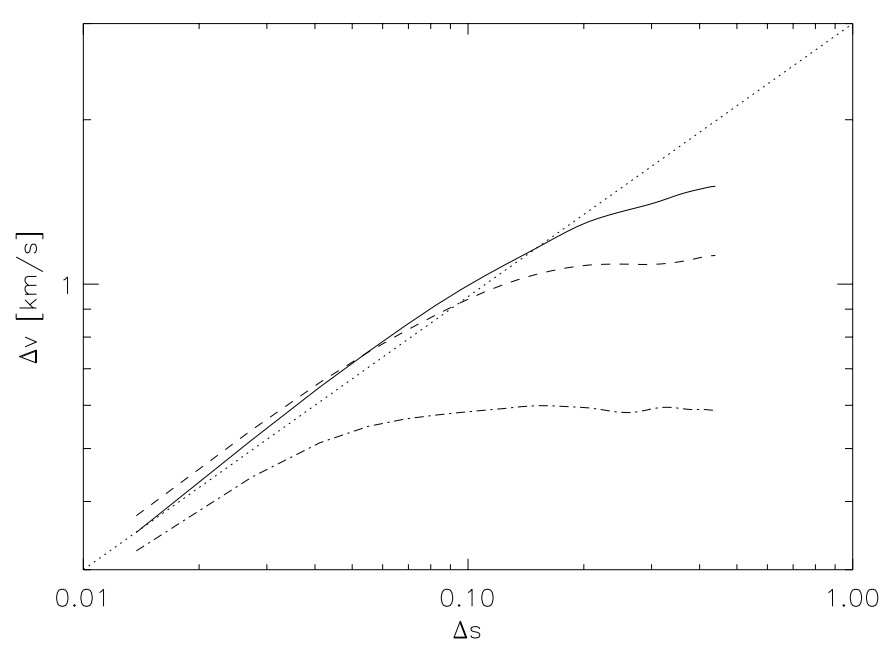

Fig. 15. Standard deviation of the centroid velocity difference PDF as a function of lag, which is equivalent to the structure function, normalised to the square root of the lag for 3D velocity cubes of a hydrodynamic simulation driven at wavenumbers of $k_{\mathrm{d}}=2$ (solid), $k_{\mathrm{d}}=4$ (dashed), and $k_{\mathrm{d}}=8$ (dot-dashed). These are models HE2, HE4, and HE8 from Mac Low (1999).

we estimate in Sect. 3.2.2 a typical width ratio between 1.5 and 1.6 for a depth comparable to the lateral extension. In Fig. 14, showing a very high $M_{0}$ simulation, the ratio is 1.5 , but for the majority of simulations we find ratios of over 1.8, suggesting that the Polaris Flare observations are of a region containing hypersonic turbulence with $M_{0}$ of order 10 or higher.

For most model PDFs we have tested three possible fits to the distribution: Gaussians fitting either the whole distribution or only the wings, and an exponential fit. Although exponential wings cannot be ruled out completely, the Gaussian fits are clearly better. We have found this to be true for all models of driven and decaying turbulence discussed here, as can be seen by visual inspection of Figs. 11 to 14 . This result agrees with the Gaussian velocity PDFs found by Chappell \& Scalo (1999) for models of decaying Burgers turbulence.

\subsection{Velocity difference PDFs}

\subsubsection{Second moments: Structure function}

In Sect. 3.3 we have shown that the second moment of the centroid velocity difference PDFs, or equivalently the structure function, is a differential measure for the size-linewidth relation, so that the same power law was found for the two functions when applied to the observations. In Fig. 15 we plot the structure function of the three models driven at different scales whose size-linewidth relation was shown in Fig. 9.

Comparison of the two figures demonstrates that the structure function provides a better estimate of the driving scale as its curvature is restricted to a narrower range around the driving scale than the curvature of the size-linewidth relation. On the other hand, only the latter indicates the dissipation scale by curvature at small lags. The different sensitivity of the two functions is caused by the different shape of their effective filter functions (Sect. 3.5). We expect that with sufficient

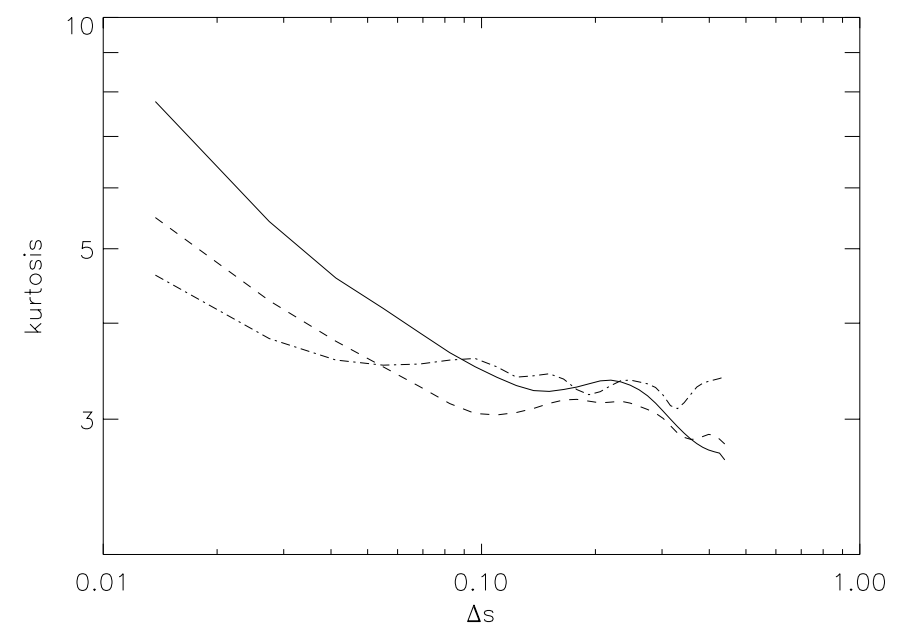

Fig. 16. Kurtosis of the centroid velocity difference distribution for a hydrodynamic simulation driven at scales of $k_{\mathrm{d}}=2$ (solid), $k_{\mathrm{d}}=4$ (dashed), and $k_{\mathrm{d}}=8$ (dash-dot). These are models HE2, HE4, and HE8 from Mac Low (1999).

dynamic range there would be a set of lags where both functions would show power-law behaviour, but our $128^{3}$ models have such limited dynamic range that there is effectively no scale where this is true. The combined set of observations, on the other hand, does have enough dynamic range to be dominated by the power-law slope, so that both functions agree in most parts of the spectrum.

\subsubsection{Fourth moments: Kurtosis}

The kurtosis of the centroid velocity difference distribution can measure correlations in the motion at certain scales. Values around three indicate Gaussian distributions, implying motions uncorrelated relative to the overall velocity field of the map considered. Miesch et al. (1999) found a decay of the kurtosis from small to large scales roughly proportional to the square root of the scale down to a value of three at the largest scales in their maps. In Sect. 3.3 we found somewhat shallower slopes, and a flat Gaussian section at scales above the total cloud size in the CfA Polaris Flare map.

In Fig. 16 we test how different driving scales in a driven hydrodynamic model influence the resulting kurtosis plots. We clearly see that the models driven at larger scales reach the Gaussian value of kurtosis at larger lags, about a factor of two below the peak size of the driving structure indicated by the $\Delta$-variance. This suggests that motion at lags above the scale of the driving process remains uncorrelated. At smaller lags, our uniformly driven models show kurtosis following $\Delta s^{-1 / 3}$, similar to the behaviour observed for the Polaris Flare maps in Sect. 3.3. None of our models reached the kurtosis values around 50 shown by the observations of Miesch et al. (1999), suggesting that additional physics, especially in the driving function, may be responsible for these high values.

Varying the resolution of our simulations does not appear to markedly change the peak value of kurtosis, although the scale at which that value is reached is always the smallest scale of the simulation, suggesting that the correlated motions 


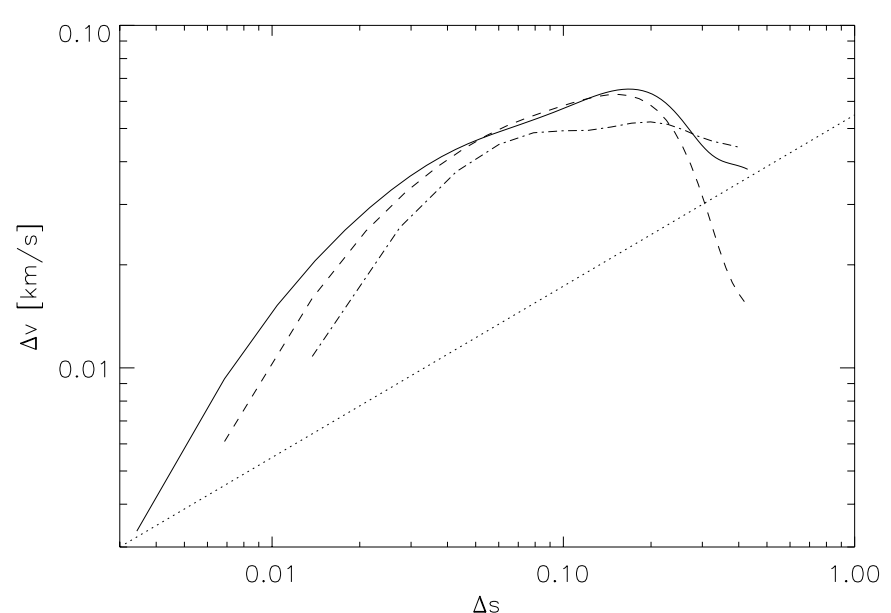

Fig. 17. $\Delta$-variance for the velocity centroids for 3D velocity cubes of a hydrodynamic simulation of decaying turbulence at time $t=0.75 t_{\text {cross }}$, computed at resolutions of $64^{3}$ (dot-dashed), $128^{3}$ (dashed), and $256^{3}$ (solid). These are models B, C, and D from Mac Low et al. (1998).

introduced by dissipation are a major influence in producing non-Gaussian velocity-difference PDFs. Magnetic fields do change the shape of the velocity difference PDF, though not drastically. Increasing field strength increases the peak value of kurtosis by about $30 \%$.

\section{4. $\Delta$-variance}

In Paper I we used the $\Delta$-variance to investigate the spatial scaling of turbulent density structure. Here, we use this method to investigate the velocity structure. In contrast to our analysis of the observations, we have access to the full 3D structure in the simulations.

We start by studying how changes in the resolution, and so in the dissipation length scale, appear in the $\Delta$-variance spectrum. In Fig. 17 we compare models of decaying turbulence that are statistically identical, but were computed with resolutions of $64^{3}$ (dash-dotted), $128^{3}$ (dashed), and $256^{3}$ (solid). The variations at large scales $(\Delta s>0.1)$ are most likely due to statistical fluctuations rather than the changes in resolution. The result of systematically increasing the resolution can be seen at small scales, as the spectrum reaches smaller scales at higher resolution. The shape of the spectrum at the smallest scales in each model is very similar, with only the scale changing. This shows the range over which numerical diffusion is acting. Above that scale, the models agree fairly well in a region that can be considered to be the inertial range of the turbulence. We see that, equivalent to the density structure, numerical diffusion causes a steepening of the spectrum, and that this reaches to a scale of roughly ten zones in every model.

Now we can consider the effects of different driving wavelengths. Figure 18 shows the results of the analysis for three hydrodynamic models driven at different scales (the $\Delta$-variance for the density distribution in these models is shown in Fig. 7b of Paper I). We plot the square root of the $\Delta$-variance, as that is the quantity directly related to the linewidth. We see pronounced peaks close to the driving scale at $0.5-0.6 \lambda_{\mathrm{d}}$. They

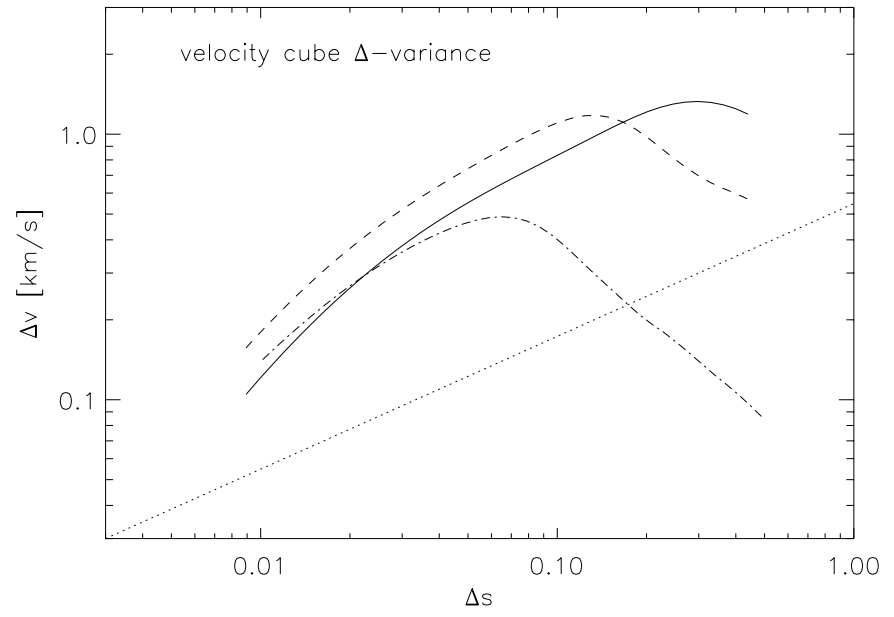

Fig. 18. $\Delta$-variances computed for $3 \mathrm{D}$ velocity cubes of a hydrodynamic simulation driven with wavenumber $k_{\mathrm{d}}=2$ (solid), $k_{\mathrm{d}}=4$ (dashed), and $k_{\mathrm{d}}=8$ (dot-dashed). These are models HE2, HE4, and HE8 from Mac Low (1999).

are even somewhat more pronounced than in the density structure of these models (Paper I), because the driving process itself is implemented in velocity space (Mac Low 1999). Below the driving scale we find power-law behaviour with a slope of 0.57 for $\Delta v$ corresponding to a slope of 1.14 for the $\Delta$-variance, down to the scale of the numerical viscosity at about ten pixels ( $\Delta s \approx 0.04$ in the figure).

How does this slope compare to the expected power spectrum? As discussed in Paper I, there is a theoretical relation between the slope of the $\Delta$-variance $\alpha$ and the index $\zeta$ of an $n$ dimensional power spectrum $P(\boldsymbol{k}) \propto|\boldsymbol{k}|^{-\zeta}$ given by $\alpha=\zeta-n$. Please note the difference to the index $\zeta_{\text {int }}$ of the often used one-dimensional power spectrum $P(k)$ that is obtained by angular integration of $P(\boldsymbol{k}), \zeta=\zeta_{\text {int }}+n-1$. Hence, the slope is translated into values of 4.14 and 2.14 for $\zeta$ and $\zeta_{\text {int }}$, respectively. Incompressible Kolmogorov-type turbulence is characterised by values of $11 / 3$ and $5 / 3$, respectively, while shockdominated velocity fields should show the Fourier transform of a step function, i.e. values of 4.0 and 2.0 , respectively. The results from the simulations are quite close to the behaviour of shock-dominated gas, but the small deviation might ask for further investigation.

For the density structure, we found $\Delta$-variance slopes between 0.45 and 0.75 in projection, corresponding to $\zeta$ (not $\zeta_{\text {int }}$ ) values between 2.45 and 2.75 , considerably flatter than the slope in the velocity structure found here. We speculate that this is due to the compressibility of the isothermal gas modelled here. The gas piles up in thin sheets and filaments, so that the density structure has behaviour somewhere in between that of a $\delta$ function and a step function. The Fourier transform of a one-dimensional $\delta$ function in the density cube is characterised by $\zeta=2$. The velocity on the other hand, remains uniform for longer distances behind shocks, so that its steeper spectral slope approaches more closely the value expected for a box full of pure step functions.

We can compare the 3D $\Delta$-variance spectrum to that from simulated velocity centroid maps. In Fig. 19 we show the 


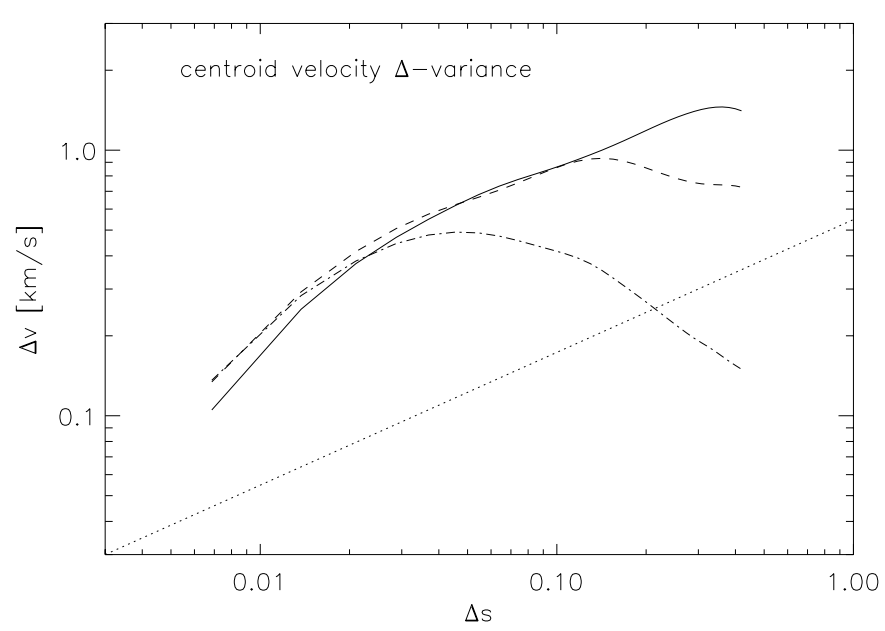

Fig. 19. $\Delta$-variances of the centroid velocity maps of the three models from Fig. 18, with driving wavenumber $k_{\mathrm{d}}=2$ (solid), $k_{\mathrm{d}}=4$ (dashed), and $k_{\mathrm{d}}=8$ (dot-dashed).

$\Delta$-variance plots for the centroid velocity maps from the models shown in Fig. 18. Here, identification of the driving scale is no longer so easy. Nevertheless, the general functional behaviour is similar. The broad maximum is shifted depending on the driving wavelength, and there is a power law range with the slope close to 0.5 . Thus, the $\Delta$-variance of the velocity centroids does seem to reflect the true cloud velocity structure, if not as clearly as the 3D spectrum. In the centroid velocities we measure the same drift index $\alpha$ as in the 3D data cube, in contrast to the density structure where the integrating projection to two dimensions preserves the power spectral exponent $\zeta$, and therefore increases the drift index $\alpha$ by 1 (Stutzki et al. 1998). This conclusion also holds for the other models so that we restrict ourselves here to the 3D $\Delta$ variance, noting that the observational centroid maps do not reveal the structural properties as clearly as discussed here.

Comparing Fig. 19 with Figs. 9 and 15 or Fig. 17 with Fig. 10, respectively, we see that the $\Delta$-variance of the velocity centroids resolves both the driving scale and the dissipation scale most clearly. Although the structure function also shows the driving scale by a change of the slope from 0.5 to 0 , the bending from 0.5 to about -0.5 in the $\Delta$-variance (Fig. 19) can be detected more easily in noisy data. The steepening at the dissipation scale which is also visible in the size-linewidth relation by a change of the slope from 0.5 to about 0.8 is also greatest for the $\Delta$-variance where a slope of about 1.2 is measured at the smallest scales.

In Fig. 20 we show the $\Delta$-variances for the $3 \mathrm{D}$ velocity structure in a sequence of four time steps in the evolution of strongly magnetised $\left(v_{\mathrm{A}} / c_{\mathrm{s}}=10\right)$, decaying turbulence. The magnitude of the variance drops over time, starting from small scales, resulting in an increase in the effective peak of the driving function and a slight increase in the effective slope of the spectrum over time. This effect was also seen in the density structure in Paper I, and there shown to be proportional to $t^{1 / 2}$ as predicted by Mac Low (1999).

To demonstrate the effect of magnetic fields we show in Fig. 21 the 3D $\Delta$-variance plots observed parallel and perpen-

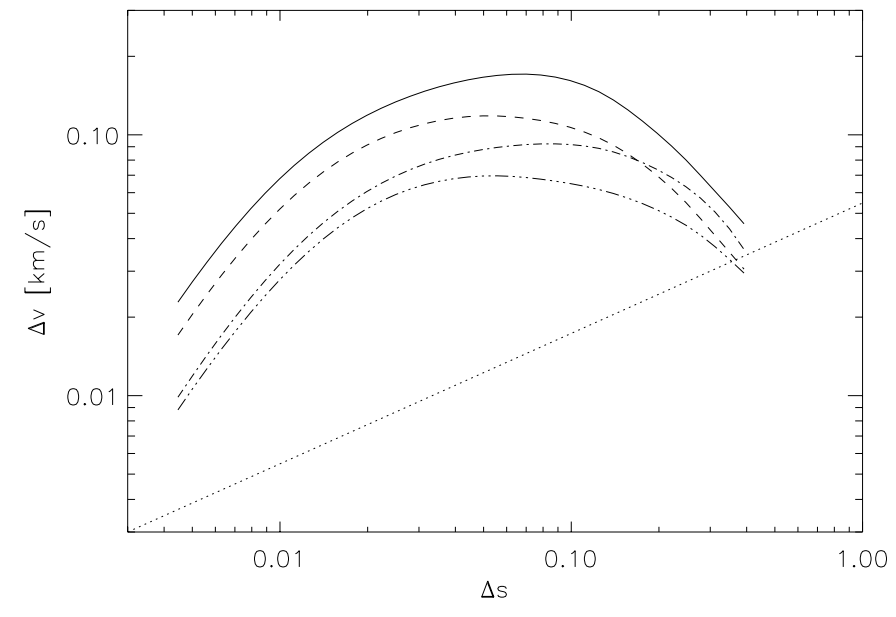

Fig. 20. $\Delta$-variance of the $3 \mathrm{D}$ velocity structure in a decaying MHD turbulence model at times of $0.5,0.75,1.5$, and 2.5 initial crossing times.

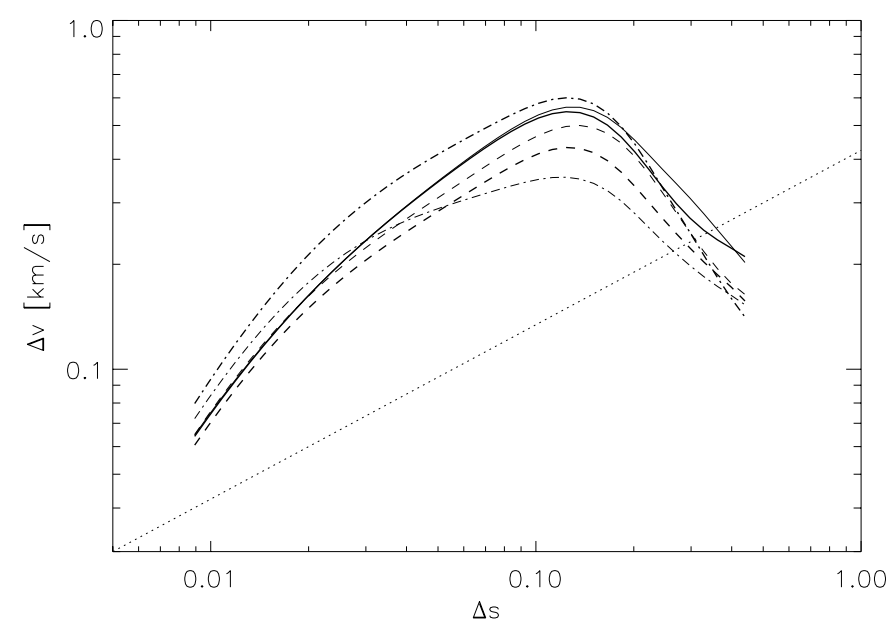

Fig. 21. $\Delta$-variance of the $3 D$ velocity structure observed parallel (thick lines) and perpendicular (thin lines) to the magnetic field for a models driven with $k_{\mathrm{d}}=4$ and no field (solid), weak field $\left(v_{\mathrm{A}} / c_{\mathrm{s}}=1\right.$, dashed), and moderately strong field $\left(v_{\mathrm{A}} / c_{\mathrm{s}}=5\right.$, dash-dot).

dicular to the initial magnetic field in two MHD simulations with different field strengths, compared to a corresponding hydrodynamic simulation. We find a substantial anisotropy in the case of a strong magnetic field (as in Sect. 5.2.1), in contrast to the hydrodynamic simulation and weak field cases. This is visible in the figure which shows a velocity variance for the strongfield case (dash-dot curves) substantially greater when viewed parallel to the initial field direction (thick line) than perpendicular to it (thin line).

The theory of incompressible MHD turbulence (Sridhar \& Goldreich 1994; Goldreich \& Sridhar 1995; Goldreich \& Sridhar 1997) predicts that the perpendicular cascade will be more efficient than the parallel cascade, draining energy more quickly from perpendicular motions and producing anisotropic wavevectors aligned parallel to the field. Our results suggests that this continues to be valid in the compressible regime. Further support for this conclusion may be drawn from the elongation of the density structures also seen in the simulations (see Fig. 5 in Mac Low 1999). 
In the weak field case (dashed line) the anisotropy is far smaller, and indeed reversed, with perpendicular motions having slightly greater power than parallel motions. However, in this case, unlike the strong field case, the field is weak enough that it is carried by the flow into a tangled geometry, so that the initial field direction no longer describes the geometry of the field well. The small anisotropy observed is only a stochastic effect of the particular field configuration at that time. In fact, the plotted model is the only example from our simulations where we find this reversal even at the low field strengths.

Aside from the anisotropy, these driven models show little overall difference in the velocity structure between a hydrodynamic model and the corresponding MHD models. A small increase in the amount of short-wavelength structure is seen with increasing magnetic field, as in the density structure (Paper I), although there is no substantial shift of the effective driving scale. The small magnitude of this effect supports the claim by Heitsch et al. (2000) that the transfer of energy to smaller scales is insufficient to support the smaller scales against gravitational collapse if they were able to collapse in the absence of magnetic fields.

\section{Physical parameters}

\subsection{Observations}

The velocity structure in the Polaris Flare maps shows basically a power-law behaviour below a few parsecs. At the smallest scales we find some indication for a steepening of the $\Delta$ variance spectrum of the velocity centroids, but the noise level in the data prevents a definite conclusion here. A similar scaling behaviour was demonstrated by Bensch et al. (2001a) for the intensity maps, reflecting the density structure. The $\Delta$-variance spectrum in the intensity maps gradually steepens from a slope $\zeta=2.6$ on large scales to $\zeta=3.3$ on the smallest scales. However, this behaviour is only visible when combining results from multiple instruments with different resolutions. The maps taken with each individual instrument appear to show only a power law within their limited dynamic range. The sizelinewidth relation and structure function show more constant slope, as is expected from their lesser sensitivity to the driving scale.

The velocity centroid PDFs show nearly Gaussian wings, as do the average line profiles, though with different widths. The PDFs of velocity difference as a function of lag, on the other hand, show non-Gaussian behaviour, with kurtosis values in some cases exceeding the exponential value of six at the smallest scales, indicating correlated motion on these scales.

We discuss below the physical implications of these observations, as deduced from our models.

\subsection{Model properties}

The clearest result we can draw from the models is that selfsimilar, power-law behaviour in both velocity and density structure can be found between the dissipation scale and the driving scale even for the highly compressible turbulence that we model here. The behaviour is less clear but still definitely present after projection into 2D. The strength of the magnetic field also does not strongly influence this conclusion, although stronger magnetic fields modify the slope of the density spectrum and do introduce measurable anisotropy into the turbulence, as shown in Fig. 21.

Above the driving scale, a flattening or turnover of the spectrum is apparent in all the measures we have studied, though it is most pronounced in the $\Delta$-variance spectra. Conversely, numerical dissipation in the models causes a clear steepening of the spectrum as structure at smaller scales disappears. Although physical dissipation will not have the same detailed properties as numerical dissipation, the general steepening of the spectrum will certainly occur. Similar behaviour is observed in incompressible turbulence (Lesieur 1997).

The velocity centroid PDFs have kurtosis values in the range $2-4$. The lowest values are distinctly sub-Gaussian, and appear to be produced only by driving at the largest available scales. We agree with Klessen (2000) that these non-Gaussian PDFs may be due to undersampling of a Gaussian distribution. No other physics that we have introduced produces such deviations. Observations of sub-Gaussian velocity centroid PDFs thus is consistent with driving from scales larger than the observed region.

The models driven at the largest scale show marked superGaussian behaviour in their velocity difference PDFs at short lags, as reflected in kurtosis values exceeding even the exponential value of six. The occurrence of such values is thus a strong indication that the primary driving occurs on scales as large as or larger than those in the map.

\subsection{Turbulence properties}

Comparison between the observations of the Polaris Flare and the turbulence simulations constrains the mechanisms driving the turbulence in this cloud. Any mechanism that drives at an intermediate length scale, such as jets from embedded protostars, should produce characteristic features in the $\Delta$-variance at that scale, which are not observed. The approximately selfsimilar, power-law behaviour seen in the observations is best reproduced by models where the energy is injected at large scales and dissipated at the small scales. The slight steepening of the $\Delta$-variance spectra of the intensity structure, which seems also to be present in the velocity structure, is consistent with turbulence models where the turbulence was recently injected and is decaying now due to the dissipation (see Paper I).

The large driving scale argues against protostellar outflows being the main driving mechanism. An attractive alternative is driving by interactions with superbubbles and field supernova remnants (e.g. Mac Low et al. 2001; Avillez et al. 2000; Norman \& Ferrara 1996). The Polaris Flare molecular cloud lies in the wall of a large cylindrical structure representing one of the nearest H I supershells, the North Celestial Polar Loop (Meyerdierks et al. 1991), adding additional support to this proposal.

The kurtosis of the velocity difference PDFs in the Polaris Flare observations, especially at small and intermediate scales, reaches values close to 10 , arguing for driving from scales 
larger than the maps on which the high kurtosis appears, adding further support to the interpretation given above. We note that many of the actively star-forming regions observed by Miesch et al. (1999) have even higher kurtosis values at small scales, suggesting that the additional physics of strong self-gravity and local heating may produce additional effects that must be examined in future work.

The dominant physical mechanism for dissipation in molecular clouds was first shown by Zweibel \& Josafatsson (1983) to be ambipolar diffusion. Klessen et al. (2000) showed that the length scale on which ambipolar diffusion will become important can be found by examining the ambipolar diffusion Reynolds number

$R_{\mathrm{A}}=\mathcal{M}_{\mathrm{A}} \tilde{L} v_{\mathrm{ni}} / v_{\mathrm{A}}$

defined by Balsara (1996) and Zweibel \& Brandenburg (1997), where $\tilde{L}$ and $\mathcal{M}_{\mathrm{A}}$ are the characteristic length and Alfvén Mach number, $v_{\mathrm{ni}}=\gamma \rho_{\mathrm{i}}$ is the rate at which each neutral is hit by ions, and $v_{\mathrm{A}}^{2}=B^{2} / 4 \pi \rho$ approximates the effective Alfvén speed in a mostly neutral region with total mass density $\rho=\rho_{\mathrm{i}}+\rho_{\mathrm{n}}$ and magnetic field strength $B$. The coupling constant depends on the cross-section for ion-neutral interaction, and for typical molecular cloud conditions has a value of $\gamma \approx 9.2 \times 10^{13} \mathrm{~cm}^{3} \mathrm{~s}^{-1} \mathrm{~g}^{-1}$ (e.g. Smith \& Mac Low 1997).

Setting the ambipolar diffusion Reynolds number $R_{\mathrm{A}}=1$ yields a diffusion length scale of

$$
\begin{aligned}
L_{\mathrm{D}} & =v_{\mathrm{A}} / \mathcal{M}_{\mathrm{A}} v_{\mathrm{ni}} \\
& \approx(0.041 \mathrm{pc}) \mathcal{M}_{\mathrm{A}}\left(\frac{B}{10 \mu \mathrm{G}}\right)\left(\frac{10^{-6}}{x}\right)\left(\frac{10^{3} \mathrm{~cm}^{-3}}{n_{\mathrm{n}}}\right)^{3 / 2}
\end{aligned}
$$

with the ionization fraction $x=\rho_{\mathrm{i}} / \rho_{\mathrm{n}}$ and the neutral number density $n_{\mathrm{n}}=\rho_{\mathrm{n}} / \mu$, with $\mu=2.36 m_{\mathrm{H}}$. If the ionization level in the Polaris Flare is low enough and the field is high enough, this length scale of order $0.05 \mathrm{pc}$ would be directly resolved in the IRAM observations. We cannot yet unambiguously say whether the observed velocity spectra show a steepening there, similar to the downturn at the dissipation scale in the numerical models. If better observations do show such a downturn in the future, that will be an indication of the dissipation scale.

\section{Summary}

We have applied several methods to characterise the velocity structure observed in the Polaris Flare molecular cloud over scales ranging from $0.015 \mathrm{pc}$ to about $20 \mathrm{pc}$. We then applied the same methods to a large suite of computational hydrodynamic and MHD models of supersonic, isothermal turbulence. The comparison between the observations and models with different parameters allows us to draw conclusions both about the properties of the analysis methods and the physical state of the observed region:

- By measuring the average variation of velocity centroids as a function of the size of a virtual scanning beam in the observations and in simulated observations, we recover
Larson's (1981) size-linewidth relation at scales where the turbulence shows an inertial range. This provides a method for measuring this relation that does not rely on the identification of isolated clumps of gas. Comparing this variation with the corresponding variation of the average total linewidth in the virtual beams allows estimation of the depth of the cloud along the line of sight.

- The spectrum of the $\Delta$-variance, a multi-dimensional wavelet transform (Stutzki et al. 1998), shows deviations from inertial scaling behaviour at the scales of driving, dissipation, observational noise, and the telescope beam more clearly than other methods that we tested, because its effective filter function is better confined in the spatial frequency domain. However the method currently lacks intensity weighting, so that it is not reliable on observed maps with large empty areas.

- The structure function characterizing the second moments of the velocity difference PDFs duplicates information recovered by the scanning-beam size-linewidth relation or the $\Delta$-variance of centroid velocities. The fourth moments measure correlated motion within a map.

- We compare two proposed methods for measuring velocity PDFs by comparing their results to the known PDFs in our models. The average line profile, determined in an optically thin line, is a better measure than the distribution of line centroid velocities. The width ratio of the two distributions depends on the depth of the observed region, as well as the Mach number of the flow, allowing us to infer a Mach number exceeding 10 for the Polaris Flare. Sub-Gaussian velocity centroid PDFs are mainly produced by the cosmic variance in the case of large-scale driving. The influence of magnetic fields and driving strength appears weak.

- The power spectrum slope $\zeta$ can be derived from the slope of the $\Delta$-variance spectrum obtained for the velocity centroid maps of the Polaris Flare. At intermediate scales in the KOSMA observations we find a slope in between 0.36 and 0.46 , corresponding to $\zeta=3.7 \ldots 3.9$, while at small scales in the IRAM map we find $\zeta=3.6 \ldots 4.4$. Models of driven, supersonic turbulence show inertial range slopes of $\zeta=3.9-4.2$, with slopes steepening to $\zeta>5$ in the dissipation range. (Dissipation is due to numerical viscosity in these models). The large error bars in the observed velocity spectra do not yet allow to draw a definite conclusion on a steepening in the dissipation range as seen in the models nor on a gradual steepening across the full range of scales as seen by Bensch et al. (2001a) in the intensity maps of the Polaris Flare. Future observations could do this.

- The observations show super-Gaussian velocity difference PDFs at small scales. Only driving at the largest scales in our models produces strongly super-Gaussian velocity difference PDFs at small lags.

- The observed structure is consistent with hydrodynamic or MHD supersonic turbulence showing a complete spectrum from a driving scale at larger than $10 \mathrm{pc}$, through an inertial range, to a dissipation scale under $0.05 \mathrm{pc}$. The combination of the density and the velocity scaling behaviour indicates a medium dominated by shocks at intermediate length 
scales creating thin sheets or filaments. Ambipolar diffusion could explain the dissipation.

- The main uncertainties come from the noise in the observational data leading to large error bars in the velocity scaling functions and the unknown anisotropy of the velocity field. Anisotropic velocity fields could be produced by large-scale driving by supernovae or by strong magnetic fields but cannot be detected in observations bound to the plane of the sky.

Acknowledgements. We thank J. Ballesteros-Paredes, D. Balsara, F. Bensch, A. Burkert, R. S. Klessen, M. D. Smith, J. Stutzki, \& E. Zweibel for useful discussions, and the anonymous referee for helping us to clarify our presentation. VO acknowledges support by the Deutsche Forschungsgemeinschaft through grants SFB 301C and 494B, and M-MML acknowledges support by the NSF through a CAREER fellowship, AST 99-85392, and by the NASA Astrophysical Theory Program through grant NAG5-10103. Computations presented here were performed at the Rechenzentrum Garching of the MaxPlanck-Gesellschaft, and at the National Center for Supercomputing Applications (NCSA), supported by the NSF. ZEUS was used by courtesy of the Laboratory for Computational Astrophysics of the NCSA. This research has made use of NASA's Astrophysics Data System Abstract Service.

\section{References}

Anselmet, F., Gagne, Y., Hopfinger, E. J., \& Antonia, R. 1984, J. Fluid Mech., 140, 63

Avillez, M. A., Ballesteros-Paredes, J., \& Mac Low, M.-M. 2000, Bull. Amer. Astron. Soc., 196, 26.08

Balsara, D. 1996, ApJ, 465, 775

Bensch, F., Stutzki, J., \& Ossenkopf, V. 2001a, A\&A, 366, 636

Bensch, F., Panis, J.-F., Stutzki, J., Heithausen, A., \& Falgarone, E. 2001b, A\&A, 365, 275

Brunt, Ch. M. 1999, Turbulence in the Interstellar Medium, Ph.D. Thesis, Univ. Massachusetts

Caselli, P., \& Myers, P. C. 1995, ApJ, 446, 665

Chappell, D., \& Scalo, J. 1999, MNRAS, 310, 1

Clarke, D. 1994, NCSA Technical Report

Dubinski, J., Narayan, R., \& Phillips, T. G. 1995, ApJ, 448, 226

Evans, C. R., \& Hawley, J. F. 1988, ApJ, 332, 659

Falgarone, E., \& Phillips, T. G. 1990, ApJ, 359, 344

Falgarone, E., Phillips, T. G., \& Walker, C. K. 1991, ApJ, 378, 186

Falgarone, E., Lis, D., Phillips, T., et al. 1994, ApJ, 436, 728

Falgarone, E., Pineau Des Forêts, G., \& Roueff, E. 1995, A\&A, 300, 870

Falgarone, E., Panis, J.-F., Heithausen, A., et al. 1998, A\&A, 331, 669

Goldreich, P., \& Sridhar, S. 1995, ApJ, 438, 763

Goldreich, P., \& Sridhar, S. 1997, ApJ, 485, 680

Goodman, A. A., Barranco, J. A., Wilner, D. J., \& Heyer, M. H. 1998, ApJ, 504, 223

Hawley, J. F., \& Stone, J. M. 1995, Comp. Phys. Comm., 89, 127

Heithausen, A., \& Thaddeus, P. 1990, ApJL, 353, L49

Heyer, M. H., \& Schloerb, F. P. 1997, ApJ, 475, 173

Houlahan, P., \& Scalo, J. 1990, ApJS, 72, 133
Joulain, K., Falgarone, E., Pineau des Forêts, G., \& Flower, D. 1998, A\&A, 340, 241

Kleiner, S. C., \& Dickman, R. L. 1985, ApJ, 295, 466

Klessen, R. S. 2000, ApJ, 535, 869

Klessen, R. S., Heitsch, F., \& Mac Low, M.-M. 2000, ApJ, 535, 887

Kolmogorov, A. N. 1941, Dokl. Akad. Nauk SSSR, 30, 9

Larson, R. B. 1981, MNRAS, 194, 809

van Leer, B. 1977, J. Comput. Phys., 23, 276

Lesieur, M. 1997, Turbulence in Fluids, 3rd ed. (Kluwer Dordrecht), 245

Lis, D. C., Pety, J., Phillips, T. G., \& Falgarone, E. 1996, ApJ, 463, 623

Lis, D. C., Keene, J,. Li, Y., Phillips, T. G., \& Pety, J. 1998, ApJ, 504, 889

Mac Low, M.-M. 1999, ApJ, 524, 169

Mac Low, M.-M. \& Ossenkopf, V. 2000, A\&A, 353, 339 (Paper I)

Mac Low, M.-M., Klessen, R. S., Burkert, A., \& Smith, M. D. 1998a, Phys. Rev. Lett., 80, 2754

Mac Low, M.-M., Balsara, D. S., Avillez, M. A., \& Kim, J. 2001, ApJ, submitted

Meyerdierks, H., Heithausen, A., \& Reif, K. 1991, A\&A, 245, 247

Miesch, M. S., \& Bally, J. 1994, ApJ, 429, 645

Miesch, M. S., \& Scalo, J. M. 1995, ApJ, 450, L27

Miesch, M. S., Scalo, J., \& Bally, J. 1999, ApJ, 524, 895

Myers, P. C. 1983, ApJ, 270, 105

Norman, C. A., \& Ferrara, A. 1996, ApJ, 467, 280

Ossenkopf, V., Bensch, F., Mac Low, M.-M., \& Stutzki, J. 1999, in The Physics and Chemistry of the Interstellar Medium, ed. V. Ossenkopf, J. Stutzki, \& G. Winnewisser, GCA-Verlag Herdecke, 216

Ossenkopf, V., Bensch, F., \& Stutzki, J. 2000, in The Chaotic Universe, ed. V. G. Gurzadyan, \& R. Ruffini (World Sci.), 394

Padoan, P., Juvela, M., Bally, J., \& Nordlund, A. 1998, ApJ, 504, 300

Padoan, P., Bally, J., Billawala, Y., Juvela, M., \& Nordlund, Å. 1999, ApJ, 525, 318

Padoan, P., Juvela, M., Bally, J., \& Nordlund, ̊.. 2000, ApJ, 529, 259

Padoan, P., \& Nordlund, Å. 1999, ApJ, 526, 279

Peng, R., Langer, W. D., Velusamy, T., Kuiper, T. G. H., \& Levin, S. 1998, ApJ, 497, 842

Pety, J., \& Falgarone, É. 2000, A\&A, 356, 279

Rosolowsky, E. W., Goodman, A. A., Wilner, D. J., \& Williams, J. P. 1999, ApJ, 524, 887

She, Z.-S. 1991, Fluid Dyn. Res., 8, 143

Sridhar, S., \& Goldreich, P. 1994, ApJ, 432, 612

Stone, J. M., \& Norman, M. L. 1992a, ApJS, 80, 753

Stone, J. M., \& Norman, M. L. 1992b, ApJS, 80, 791

Stutzki, J., \& Güsten, R. 1990, ApJ, 356, 513

Stutzki, J., Stacey, G. J., Genzel, R., et al. 1988, ApJ, 332, 379

Stutzki, J., Bensch, F., Heithausen, A., Ossenkopf, V., \& Zielinsky, M. 1998, A\&A, 336, 697

Tauber, J. A. 1996, A\&A, 315, 591

Vázquez-Semadeni, E. 2000, in The Chaotic Universe, ed. V. G. Gurzadyan, \& R. Ruffini (World Sci.), 384

Vázquez-Semadeni, E., Ballesteros-Paredes, J., \& Rodriguez, L. F. 1997, ApJ, 474, 292

Zagury, F., Boulanger, F., \& Banchet, V. 1999, A\&A, 352, 645

Zweibel, E. G., \& Brandenburg, A. 1997, ApJ, 478, 563 (Erratum: $485,920)$

Zweibel, E. G., \& Josafatsson, K. 1983, ApJ, 270, 511 